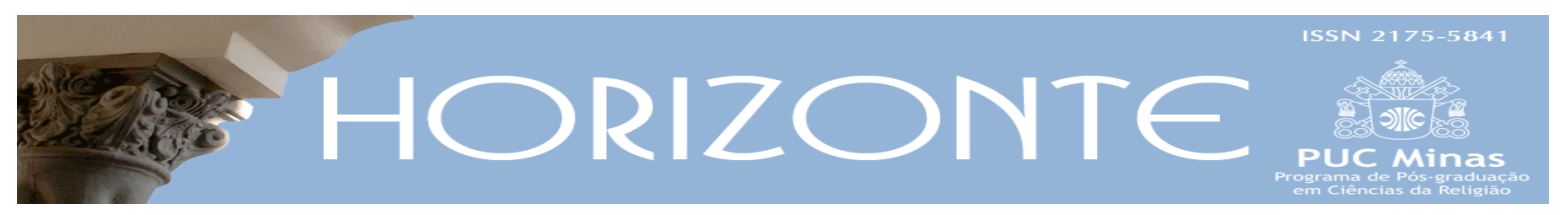

Dossiê: Ordens Religiosas Medievais: Poder e Sociedade - Articulo Originale (c) (i)

\title{
La molteplice funzione politica di un episodio agiografico: il servizio di Bernardino da Siena all'Ospedale della Scala durante la peste
}

\author{
The Multiple Political Function of a Hagiographical Episode: \\ The Bernardino da Siena Service in the Hospital of Scala during the plague
}

Pietro Delcorno *

\begin{abstract}
Riassunto
Questo contributo mette in luce la molteplice funzione politica di uno schema agiografico centrale nella rappresentazione della giovinezza di Bernardino da Siena: il suo servizio durante la peste del 1400 presso I'Ospedale di Santa Maria della Scala di Siena. La memoria di questo episodio si prestava ad essere utilizzata tanto dall'Osservanza minoritica quanto in diversi contesti locali. In questo racconto Bernardino è presentato come capace di rispondere ai bisogni della città non solo attraverso le proprie virtù, ma radunando altre persone e convincendole a impegnarsi per il bene comune, anche a rischio della propria vita. L'episodio, così come è raccontato dagli agiografi, incorpora la prima predica fatta dal giovane Bernardino (ancora laico) ai membri della confraternita dell'Ospedale. II testo indica programmaticamente la necessità per i laici di anteporre il bonum commune al bonum proprium e delinea un ruolo di leadership dei frati dell'Osservanza nel cuore delle città. Inoltre, se a Siena la memoria della peste del 1400 serviva a sottolineare il rapporto tra il santo e le istituzioni della città, la raffigurazione di tale episodio in alcuni affreschi dipinti a Lodi nel 1476 non soltanto veicolava un modello di spiritualità laicale ma serviva a promuovere e legittimare la riforma ospedaliera in corso a Lodi.
\end{abstract}

Parole Chiave: Bernardino da Siena, Agiografia, Ospedale, Peste, Opere di Misericordia

\begin{abstract}
This article highlights the multiple political function of a central episode in the hagiographical representation of the youth of Bernardine of Siena, namely his service at the hospital of Santa Maria della Scala of Siena during the 1400 plague. The memory of this episode served the purposes of the Franciscan Observance as well as those of different local contexts. This story portrayed Bernardino as able to respond to the needs of the city not only by means of his personal virtues but also by gathering other people and convincing them to work for the common good, even putting their own lives at risk. The hagiographical episode includes the first "sermon" of Bernardino, who - still as a lay man - addressed the members of the hospital confraternity. This text, programmatically, emphasises the necessity for the lay people to prioritize the bonum commune over the bonum proprium, and indirectly depicts the leadership of the Observant friars in the life of the city. Moreover, while Siena used the memory of the 1400 plague to affirm the connection between Bernardino and its civic institutions, the representation of this episode in a set of frescoes painted in Lodi in 1476 not only depicted a model for the laity but was also functional in legitimizing the reform of the hospitals in that city.
\end{abstract}

Keywords: Bernardine of Siena, Hagiography, Hospital, Plague, Works of Mercy

Articolo presentato il 12 agosto 2017 e approvato il 07 dicembre 2017.

* PhD student in Medieval History (Radboud University Nijmegen), Master Degree in Italian Linguistics and Literary Civilizations with Distinction (University of Bologna), Licence e Bachelor of Theology with Distinction (Faculty of Theology of Emilia Romagna (Bologna). Paese di origine: Italia. E-mail: p.delcorno@let.ru.nl

Horizonte, Belo Horizonte, v. 15, n. 48, p. 1354-1393, out./dez. 2017 - ISSN 2175-5841 


\section{Introduzione}

Il giorno di Pentecoste del giubileo del 1450, al termine di una memorabile cerimonia, Bernardino da Siena venne solennemente canonizzato, ricordandone anzitutto la prodigiosa attività come predicatore (PELLEGRINI, 2009). Secondo gli agiografi, Bernardino era stato sostanzialmente capace di "rifondare" con la propria azione l'Ordine minoritico e di trasformare, attraverso la sua predicazione, l'Italia intera da paese corrotto da ogni sorta di peccato a una specie di claustrum..$^{1}$ Il nuovo santo, oltre ad essere indicato quale modello per tutti i predicatori, rappresentava un riferimento per l'intero Ordine minoritico, nei suoi diversi rami. In particolare, i fratres de familia dall'Osservanza, guidati in quegli anni dall'energica azione di Giovanni da Capestrano, utilizzarono - implicitamente o esplicitamente - il santo senese in funzione «propagandistica e consolidante» (PELLEGRINI, 2004, p. 53). ${ }^{2}$

Se l'inesauribile impegno sul pulpito di Bernardino costituiva il dato più evidente, questo non fu però l'unico aspetto presente nel ricco dossier agiografico costruito in vista della sua canonizzazione e lievitato poi nei decenni successivi insieme alla diffusione capillare del suo culto (SOLVI, 2014). Accanto al predicatore, c'era spazio anche per il Bernardino laico, esemplare nella sua vita secolare prima dell'ingresso nell'Ordine. Nella stessa iconografia, si può trovare il nuovo santo raffigurato non solo sul pulpito (fig. 1), ma anche come un giovane laico, chino a servire i malati (fig. 2). Questa immagine rimanda a un precedente giubileo, quello del 1400, quando il giovane senese si era impegnato in prima persona presso l'Ospedale di Santa Maria della Scala per fronteggiare l'infuriare della peste in città. 3 Un recente convegno sui nuovi santi come modello di virtù alla fine del Medioevo è

\footnotetext{
${ }^{1}$ «Tunc est renovata Italia cum praedicationibus et quasi tota videbatur claustrum et reformata per Dei gratiam»; Giovanni da Capestrano, cit. in Quaglioni (1989, p. 126). Il concetto si trova già nella bolla di canonizzazione di Nicolò V "Misericordias domini” (24 maggio 1450).

${ }^{2}$ Si veda Caciotti \& Melli (2008); I frati osservanti, 2013; e sull'Osservanza in generale, Mixson \& Roest, (2015). Un utile quadro sul minoritismo quattrocentesco in Merlo (2003, p. 305-381).

${ }^{3}$ Preziose notizie storiche sull'Ospedale della Scala durante la peste del 1400 si ricavano da una fonte peculiare come il Libro del pellegrino (PICCINNI 2003, p. 41-46).
} 
stato l'occasione per iniziare a riflettere sulla sfaccettata costruzione agiografica della vita secolare del giovane Bernardino e su questo aspetto poco esplorato nel campo degli studi bernardiniani, ovvero: san Bernardino modello per i laici (DELCORNO, 2016). Ne è emerso un quadro dinamico, all'interno del quale veniva sottolineata la versatilità di Bernardino, presentato (anche) come laico esemplare. 4

Nella costruzione del periodo secolare della vita del santo svolge un ruolo particolare l'episodio appena menzionato, cioè il suo servizio durante la peste del 1400. Perché la memoria di quest'episodio acquista rilievo? Come viene costruita, affermata, rielaborata? Quale funzione ricopre nell'evolversi del discorso agiografico su Bernardino? Indagare la costruzione agiografica di questo specifico episodio e il suo mutare nei diversi contesti permette di riflettere, in ultima analisi, sulla molteplice valenza politica di un racconto agiografico, utilizzabile - come vedremo - tanto dai leader dell'Osservanza minoritica quanto dalle autorità cittadine. Lo studio di questo episodio permette così di osservare, quasi in forma di micro-analisi, alcuni elementi fondamentali della dinamica interazione tra i modelli di società elaborati e presentati dagli ordini religiosi e i concreti ambiti locali dove questi modelli vengono adattati alle specifiche e diverse esigenze. ${ }^{4}$ Per il contesto religioso in cui matura il giovane Bernardino, si veda Pellegrini (2016). Ringrazio la prof.ssa Pellegrini che cortesemente
mi ha inviato in anteprima il suo saggio. 
Fig. 01: Bernardino predica a L'Aquila. Gian Giacomo da Lodi. Vita di San Bernardino (1476-1477). Chiesa di San Francesco, Cappella di San Bernardino. Copyright: Pietro Delcorno

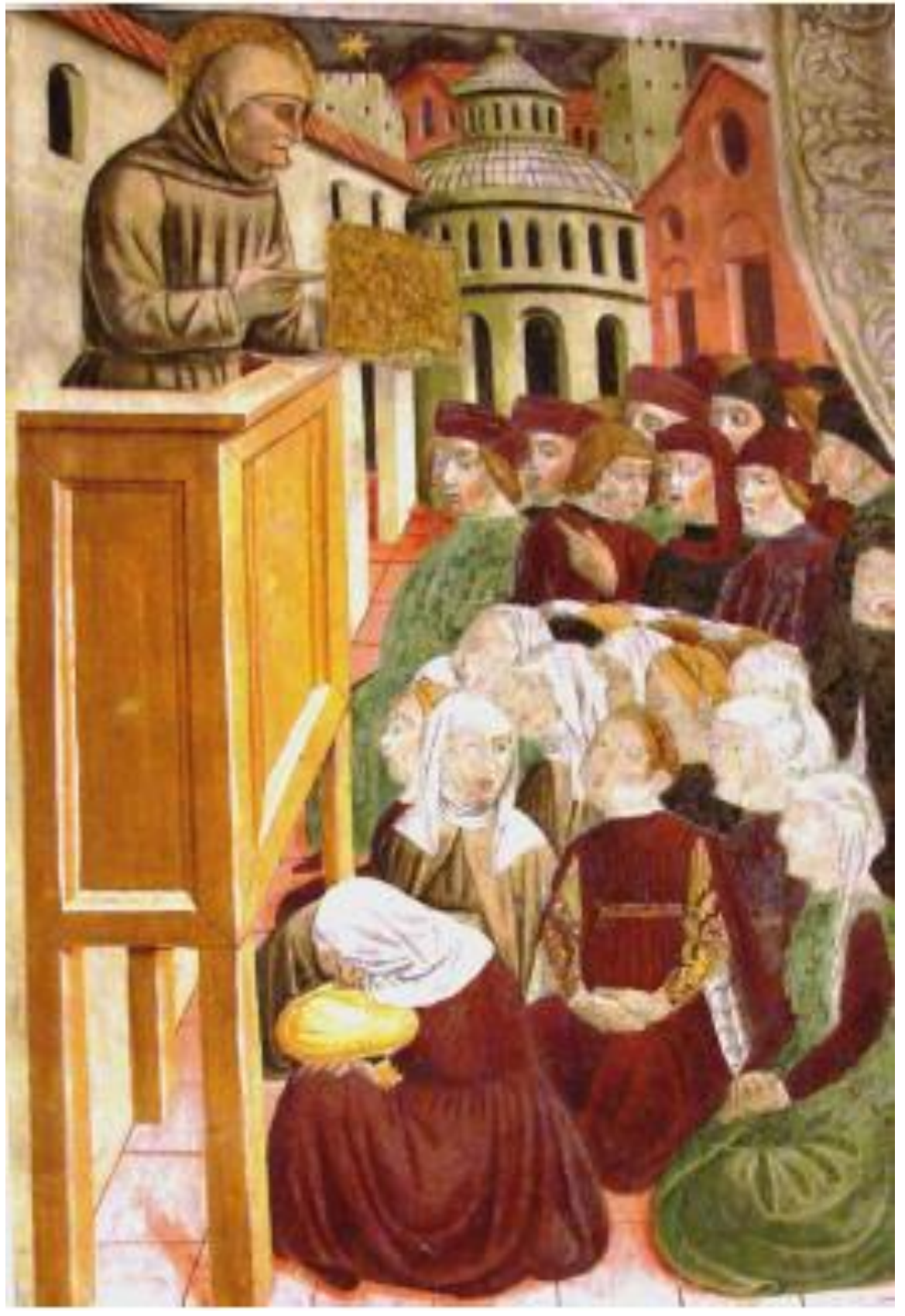


Fig. o2: Bernardino medica un malato. Gian Giacomo da Lodi. Vita di San Bernardino (1476-1477). Chiesa di San Francesco, Cappella di San Bernardino. Copyright: Pietro Delcorno

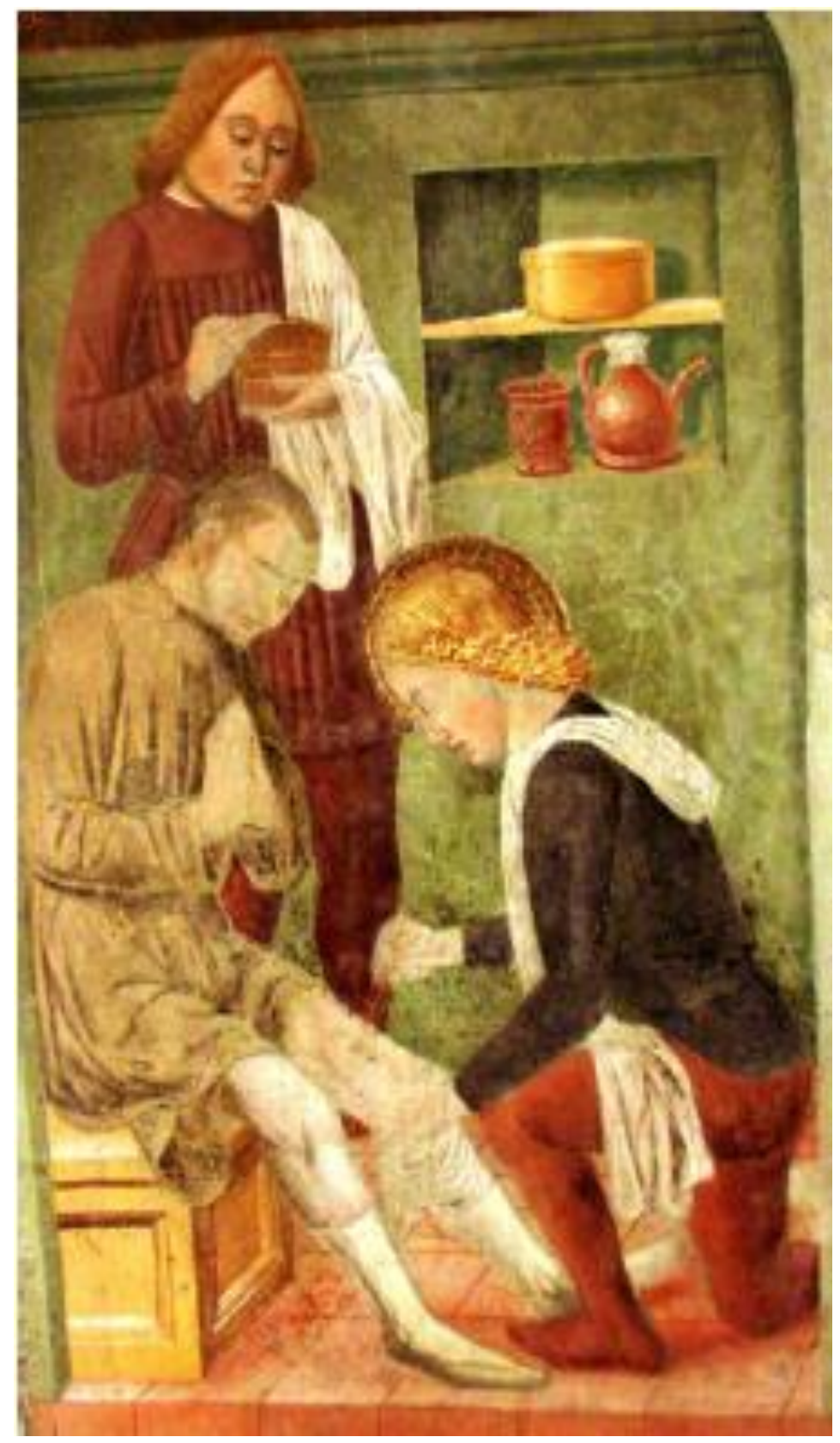




\section{Il processo di canonizzazione e la Vita di Benvoglienti}

Gli articuli interrogatori formulati per il processo di canonizzazione concentrati anzitutto nel far emergere le sue peculiarità come predicatore - dedicano poco spazio alla vita di Bernardino prima del suo ingresso nell'Ordine. L'unico episodio che si staglia con precisione è il suo servizio durante la peste del 1400. Uno degli articoli illustra in modo specifico la gravità della pestilenza, chiarisce come Bernardino si sia prodigato in questo servizio mosso solo dall'amore per i malati («frater Bernardinus, adhuc in habitu seculari degens, ad hospitale Sancte Marie de Scala de Senis vulgariter nuncupatum, nimia caritate accensus et gladio compassionis erga egrotos confossus accessit») e senza cercare alcuna ricompensa umana, ma solo a lode e gloria di Dio e della Vergine («nulla alia remunerations expectata temporali, sed tantummodo ad laudem et gloriam omnipotens Dei et beate Marie virginis premissa et alia pietatis opera exercuit»), descrivendo poi nel dettaglio i concretissimi servizi svolti dal giovane: «[...] ipsisque infirmis attente et sedulo serviebat eis cibaria ministrando, putredines et macies abstergendo, ad pacientiam exhortando, morientes de sacramentis provideri faciendo, sepulturam mortuis procurando» (PROCESSO, 2009, p. 14).

Le prime due indagini in partibus condotte principalmente a L'Aquila, dove il predicatore era morto nel 1444, raccolsero solo conferme indirette su questo punto, mentre la fase senese della terza indagine (1448) permise di registrare testimonianze dirette. C'era chi aveva condiviso con Bernardino questo servizio, come l'amico d’infanzia Pietro di Maestro Martino («Interogatus in causa sciencie, dixit quod ipse fuit socius eius in dicto hospitali et servicio, et vidit eum ibi servitia de quibus in articulo exercentem») o Guglielmo Martini, sagrestano presso l'Ospedale della Scala all'epoca della peste (PROCESSO, 2009, p. 354 e 359). Tra i compagni di Bernardino di quegli anni alcuni potevano solo confermare indirettamente quanto successo, perché fuggito dalla città, come racconta Giacomo di Andrea de Passinelli («ipse testis, propter pestem, se absentaverat»), o perché prudenzialmente mandato via dalla propria famiglia, come Giovanni Vannini («tunc temporis, propter pestem, 
misit eum pater suum ad Montem de Laterone») (PROCESSO, 2009, p. 363 e 386). Restare a Siena non era stata una scelta scontata.

Per rimediare all'iniziale mancanza di informazioni sulla giovinezza di Bernardino, già nel 1446 Giovanni da Capestrano chiese a Leonardo Benvoglienti di scrivere una Vita Bernardini ante ingressum religionis.5 Entrambi erano direttamente coinvolti nel promuovere questa canonizzazione: l'uno come vicario dell'Osservanza e regista di tutto il processo, l'altro quale personaggio di spicco della classe dirigente senese, inviato già nel luglio 1444 come ambasciatore presso la curia pontificia per sollecitare la canonizzazione del concittadino (PRUNAI, 1966). È stato anzi ipotizzato che la stesura dell'articolo sulla peste per l'indagine in partibus dipenda dalla testimonianza di Benvoglienti, presente nell'estate 1444 presso la curia romana (PIANA, 1944-1950, p. 168).

La Vita scritta da Benvoglienti è il testo fondamentale sul periodo senese e laico della vita di Bernardino. Essa venne incorporata da Giovanni da Capestrano con lievi modifiche - nella Vita presentata da questi nel 1449 al capitolo dell'Osservanza, in vista dell'imminente canonizzazione (BARTOLOMEI ROMAGNOLI, 2009, p. 6). Già durante la seconda inchiesta del processo, quella aquilana del 1447, l'importanza del testo di Benvoglienti venne sancita copiandolo tra i documenti ufficiali come una sorta di testimonianza scritta. ${ }^{6}$ Un testo agiografico - voluto e scritto da chi sta promuovendo il processo di canonizzazione - entra così a far parte di una collezione di testi giuridici, mostrando perfettamente l'interazione tra i due ambiti. Come ha ricordato Letizia Pellegrini, «dal triangolo Benvoglienti-Capestrano-atti processuali, muove tutto un filone di agiografie bernardiniane» (PELLEGRINI, 2004, p. 69).7

\footnotetext{
${ }^{5}$ Si veda la lettera di Benvoglienti a Giovanni da Capestrano (8 maggio 1446) (BENVOGLIENTI, 1902, p. 58-59). Sull'agiografia bernardiniana, oltre a quanto menzionato, si vedano: Montesano (1994); Bartolomei Romagnoli, (2002); Solvi (2009) et Checcoli (2013). ${ }^{6} / /$ processo, pp. 271-285. Benvoglienti sarà poi tra i testimoni ascoltati a Siena nel 1448 (ivi, p. 348-353).

${ }^{7}$ A partire dal 1478, la Vita di Benvoglienti venne inclusa in varie edizioni della Legenda aurea, aumentandone ulteriormente la diffusione (DELCORNO, 2016, p. 229).
} 
Facendo perno sulla devozione per la Vergine, il testo di Benvoglienti mette in luce la purezza di Bernardino e la sua carità. Quest'ultima virtù è dimostrata in particolare attraverso la dettagliata descrizione del servizio svolto, all'età di vent'anni, presso l'Ospedale della Scala. Oltre alla personale virtù del santo, questo episodio serve a delineare un Bernardino profondamente collegato alla vita civile senese, riaffermando così il legame con questa città. Tale legame rischiava infatti di passare in secondo piano a seguito della morte avvenuta a L’Aquila. La città di Siena, grazie anche all'opera di Benvoglienti, si insinuò con efficacia «nella gestione dell'immagine di Bernardino defunto e nello svolgimento del processo di canonizzazione», sottraendo così il monopolio a L’Aquila (PELLEGRINI, 2009, p. $\left.41^{*}\right)$.

Prima di descrivere la pestilenza, Benvoglienti ricorda l’ingresso di Bernardino nella Compagnia della Vergine Maria, la confraternita di flagellanti che si riuniva nei locali dell'Ospedale della Scala. Questa confraternita è presentata quale «speculum secularium devotionum» e vera fucina di santi, tra i quali si ricordano Pietro Petroni, Andrea Gallerani, Giovanni Colombini e i primi Gesuati. Benvoglienti associa così Bernardino a una precisa tradizione di santità senese e a una prestigiosa istituzione della città, l'Ospedale della Scala, in quegli anni al centro di un vasto programma di lavori per esaltarne la funzione sia assistenziale sia di rappresentanza civile. Tra il 1439 e il 1445, la costruzione del pellegrinaio e della sagrestia e il loro innovativo programma iconografico erano serviti a riaffermare la funzione civile dell'ospedale e a rafforzare l'immagine stessa della città. Questa istituzione si mostrava infatti con orgoglio quale «impresa della pubblica carità» di Siena (PICCINNI, 2003 et SORDINI, 2011). Già nel 1445, il Vecchietta - su precisa commissione - aveva inserito Bernardino tra i santi e beati senesi raffigurati nella sagrestia dell'Ospedale della Scala sull'Arliquiera, cioè l'armadio che custodiva le reliquie più preziose della città e il punto focale delle connesse generose indulgenze concesse nel 1443 da Eugenio IV. In questo modo, immediatamente dopo la sua morte, Bernardino venne incastonato nel pantheon della santità cittadina e nel cuore sacro dell'Ospedale della Scala. Il suo crescente culto locale trovò così in 
quest'istituzione uno dei punti di riferimento, come attesta la registrazione dei miracoli nel processo di canonizzazione (NORMAN, 2012).

Descrivendo la pestilenza, Benvoglienti non si limita a esaltare l'eroica carità di Bernardino, ma allarga il discorso a una dimensione corale, descrivendo il carisma del giovane senese, capace di coinvolgere altre persone e di organizzare l'ospedale durante l'emergenza. Stando al racconto, la peste si abbatte con violenza sulla città colpendo in particolare il personale dell'Ospedale della Scala dove, quanto più cresce il numero dei malati, tanto più diminuisce il numero di chi li assiste («et cum maior ibi necessitas incumbebat ministerii, magis locus erat derelictus»), anche perché non si trova più nessuno disposto a rimpiazzare chi è morto, neanche a prezzo di un lauto compenso. Solo il giovane Bernardino, ispirato da Dio, si rende disponibile a tale compito, mosso unicamente dall'amore per Cristo e per il prossimo ( «sponte mortem subire exponens, ut Christo in pauperibus suis ministret»). Dopo alcune settimane di servizio, egli si rende conto che le sue forze non bastano a far fronte alla mancanza di inservienti. Radunati allora dodici compagni della confraternita, li esorta a unirsi a lui nel servizio ai «pauperes Christi infirmos». Si tratta di un punto di grande rilievo nell'economia del testo di Benvoglienti, perché questo diventa il primo e unico sermone pronunciato da Bernardino in questa Vita. L'agiografo prima riassume le argomentazioni presentate da Bernardino e poi, improvvisamente, passa al discorso diretto, quasi a volere far sentire la voce stessa del santo. In questa esortazione viene anzitutto richiamato il fondamento stesso delle opere di carità, cioè che «chi serve il povero di Cristo, serve Cristo stesso», assimilando la possibilità di morire servendo gli appestati e la piena conformazione a Cristo «che è morto per noi». Bernardino menziona poi in rapida successione le immagini bibliche della distruzione di questo mondo («già brucia questo mondo, la scure colpisce l'albero, la falce miete il raccolto...») per poi smascherare come tutti loro, vedendo morire giorno dopo giorno amici e compagni, vivano nell'incertezza della propria sorte. In questa prospettiva, porsi a servizio degli infermi non costituisce una follia ma un saggio investimento. Se nessuno può dirsi al sicuro durante la pestilenza, morire servendo i poveri vorrebbe dire «volare presso Dio»; chi invece sopravvivrà si potrà rallegrare per tutta 
la vita di quanto ha fatto perché «nessuna opera buona resta senza ricompensa presso Dio». Per questo, conclude il giovane Bernardino, «qualunque cosa ci accadrà, sia che viviamo sia che moriamo, servendo Dio guadagniamo». Ecco che la carità diventa un investimento remunerativo: «Deo serviendo lucramur». 8

Nel racconto di Benvoglienti, Bernardino è descritto così come capace di convincere e coinvolgere i compagni. La sua leadership inoltre è ufficialmente riconosciuta dal rettore di Santa Maria della Scala che gli consegna le chiavi dell'ospedale. Bernardino non solo compie personalmente tutte le opere di misericordia, minutamente descritte nel racconto (dall'accogliere i pellegrini al seppellire i morti) ma guida anche gli altri in questa impresa di carità. Emerge chiaramente la dimensione civica del racconto elaborato dal diplomatico senese. L'Ospedale della Scala era uno dei simboli identitari della città di Siena e il suo "biglietto da visita", come mostrano i famosi affreschi del pellegrinaio, dipinti tra il 1441 e il 1444. In essi si mostra la raffigurazione ideale dell'efficiente caritas senese, l'autocomprensione della città, con una valenza politica e una funzione di propaganda paragonabile - con le dovute misure - al famoso affresco del buon governo dipinto un secolo prima da Ambrogio Lorenzetti (SORDINI, 2011).

Nella prospettiva di Benvoglienti, la drammatica situazione dell'ospedale durante la peste, quando nessuno sembra disposto a servire gli ammalati, non rappresentava soltanto un'emergenza di tipo sanitario. In questa crisi erano a rischio anche l'identità cristiana della città e il suo prestigio. L'emblema della carità di Siena, il luogo dove generazioni di santi avevano servito poveri e ammalati, stava infatti fallendo la sua missione, mostrando così il disgregarsi del tessuto della civitas col venire meno di quella caritas, di quella solidarietà interna della comunità politica

\footnotetext{
8 «Sed cum videret ministrorum inopiam et paucitatem, in infirmorum multitudinem et necessitatem convocabit duodecim spirituales iuvenes et illos hortatus est velint una secum pauperes Christi gubernare infirmos, asserens illis quod qui pauperi Christi ministrat, Christo ministrat, et si casu in tali exercitio et ministerio quis moritur, pro Christo moritur, et quod nil optabilius esse christiano debet quam pro eo Christo mori, qui mortuus est nobis. "Simus omnes confratres, iam fere crematur mundus, securis ad arborem posita est, falx messem sollicitat. Quam de nobis habere certitudinem possumus, cum et alios cotidie crebo mori videamus et fere vivos et sanos mori quosdam videamus nobis intimos socios et amicos? $\mathrm{Si}$ in hac caritate morimur, ad Deum volamus. Si autem evademus, toto tempore nostro letabimur tale obsequium in pauperibus suis prestitisse Deo, nobis recolentes Evangelium illud, quod nullum bonum irremuneratum est apud Deum. Quocumque igitur vertamur, sive vivamus, sive moriamur, Deo serviendo lucramur"» (BENVOGLIENTI, 1902. p. 69).
} 
che avrebbe dovuto invece costituirne il fondamento ultimo. Andando oltre la virtù personale di Bernardino, in questo racconto si presenta il santo come il perfetto civis cristiano capace di risvegliare l'autentico spirito della città in una risposta collettiva. Non solo egli convince i compagni, ma il loro esempio attrae, ricompone, lega di nuovo insieme gli altri cives, come afferma Benvoglienti: «L'esempio della fervente carità di Bernardino e dei suoi compagni attirò al servizio dei malati molti altri giovani e cittadini, tra i quali non pochi morirono in quel periodo».9 $\mathrm{E}$ essenziale cogliere come in questo racconto l'aspetto "miracoloso" non sia la protezione divina per chi volontariamente assiste i malati - molti moriranno in tale servizio - ma il riattivarsi della carità nella vita della città. Una caritas che è qui eminentemente virtù politica, radicata nella riflessione della testualità francescana - e bernardiniana - in materia (EVANGELISTI, 2015). Viene qui espresso in maniera chiara l'ideale di una «carità cristomimetica e civile» - come l'ha definita Paolo Evangelisti modellata sull'esempio di Cristo che offre la sua vita per gli altri e basata sull'equiparazione tra il sacrificio dei fideles per Cristo e il sacrificio dei cives per la comunità (EVANGELISTI, 2004, p. 104-105 et EVANGELISTI 2008). La passione di Cristo diventa così icona politica per eccellenza, come appare evidente là dove Bernardino dice ai suoi compagni che «se per caso qualcuno morirà in questo esercizio e servizio, morirà per Cristo, e non vi può essere nulla di più desiderabile per un cristiano che morire per Cristo, il quale è morto per noi».10

In questo racconto, Bernardino è presentato anzitutto come «il santo laico cittadino della spiritualità confraternale, chino sulle sofferenze [...] di Siena ferita dalla peste» (BARTOLOMEI ROMAGNOLI, 2009, p. 9). Benvoglienti non propone però una lettura ingenua o riduttiva, perché chi scrive è un membro dell'élite senese, frequenta la curia di Roma e collabora con Giovanni da Capestrano, è cioè perfettamente consapevole della rete di interessi assai più ampi di quelli legati a un

\footnotetext{
${ }_{9}^{9}$ «Fervoris et caritatis exemplar Bernardini et sociorum eius quam plurimos alios iuvenes et cives attraxit ad servitium infirmorum, de quibus tamen non pauci eo tempore decesserunt» (BENVOGLIENTI, 1902, p. 64). Capestrano esplicita che si sta parlando di chi serve e non dei malati: "de quibus nonnulli in tali servitio decesserunt» (CAPESTRANO, 1635, p. XXX), là dove la frase di Benvoglienti era ambivalente.

${ }^{10}$ Vedi nota 8 per il testo latino.
} 
semplice santo locale. Piuttosto, attraverso questi episodi, il diplomatico senese compie un'operazione politica precisa, tesa a riaffermare un legame indissolubile tra il santo e la città di Siena, richiamata sia nella precisa topografia che caratterizza questa Vita, sia nell'articolazione delle sue istituzioni, prima fra tutte l'Ospedale della Scala, la cui centralità materiale e simbolica nel tessuto urbano lo rende un luogo fondamentale nell'autocoscienza cittadina. Era stato lo stesso Bernardino, predicando sul Campo di Siena, a ricordare la fama dell'ospedale e a definirlo icasticamente come «uno degli occhi» (l'altro è il Duomo) che definivano il volto della città. ${ }^{11}$ La Vita scritta da Benvoglienti, e in particolare l'episodio della peste, fa parte quindi della strategia con cui la Repubblica di Siena si impegna per costruire un culto civico che si stagli nel panorama cittadino, simbolo e strumento di una rinnovata coesione sociale (PELLEGRINI, 2016). In questo senso tutto il racconto di Benvoglienti è attento a mettere in luce le figure senesi che accompagnano l'ascesa del giovane Bernardino verso la santità, descritta come il frutto eccezionale di una consolidata e viva tradizione di pietà e carità cittadina. Parafrasando un noto slogan, si potrebbe dire che Bernardino viene presentato qui come "il più santo dei senesi, e il più senese dei santi”. Infatti, l’itinerario laicale e senese consegna ai Francescani una persona già pienamente santa, come nel racconto agiografico riconosce implicitamente fra Giovanni Ristori, al momento di accogliere Bernardino nell'Ordine. ${ }^{12}$

\section{Una possibile eco autobiografica?}

In questo racconto Leonardo Benvoglienti fa pronunciare a Bernardino un breve sermo de peste, in alcuni tratti accostabile ai sermoni modello in circolazione (HANSKA, 2002). Il testo è chiaramente costruito dall'agiografo, ma ci si può

\footnotetext{
${ }^{11}$ "lo vi ricordo che quello è uno degli occhi de la nostra città, e l'altro occhio è il Vescovado: stanno molto bene a lato l'uno all'altro. L'occhio dritto è el Vescovado, e 'l sinistro è lo Spedale: el naso è la piazza che è in mezzo. Vedi che è longhetta come è il naso. Doh, cittadini, procurate a quello Spedale! Fate che si mantenghino le limosine continuamente [...]. Da la Casa de lo Spedale è utilissima cosa la limosina che n'esce; e però vi prego, che non la lassiate mai mancare; ché io mi credo che quella sia una de le cagioni, che Idio placa I'ira sua verso di voi» (BERNARDINO, Siena,1427, p. 1206). Si veda inoltre PORTICI, 2009.

12 "Ordo nostrer vere iam diu talem receptione non fecit, ex qua maior fructus appareat, et exaltatio ordinis sicut de receptione ista hodie per nos facta de Bernardino» (BENVOGLIENTI, 1902, p. 74).
} 
chiedere se in questo episodio egli non utilizzi - come in altri punti della Vita materiale attinto dai sermoni stessi di Bernardino. Se i riferimenti espliciti alla peste del 1400 sono scarsi nei sermoni bernardiniani - torneremo più avanti su un passo del ciclo senese del 1427 - in una sezione del sermone sull'amore del prossimo presente nel Quadragesimale de Evangelio Aeterno si trova un vibrante appello al dovere morale di assistere gli appestati, presentandola quale massimo esempio di carità (BERNARDINO, Quadragesimale de Evangelio Aeternovol. 3, 1956, p. 127129). Per illustrare questo punto, Bernardino si interroga su cosa succeda se il peggiore pubblico peccatore («publicus usurarius, blasphemator iniquus, sodomita magnus, impius homicida, proditor, raptor atque latrocinibus plenus, periurus, mendax et patricida et quibuscumque aliis gravibus sceleribus irretitus»), mosso da carità, si ponga a servizio degli appestati e, servendoli in maniera esemplare, si ammali e muoia senza essersi confessato e aver fatto penitenza: «Quid, queso, de amima huius pensandum est? Quid de illo credendum? Quid sperandum? Bene, melius, optime sperandum puto!».13 Replicando al possibile stupore degli ascoltatori, Bernardino spiega che la carità verso gli appestati racchiude in sé l'intero cammino penitenziale: conformandosi alla passione di Cristo ( Si Cristus pro nobis animam suam posuit, et nos debemus pro fratribus anima ponere»; cfr. 1 Giovanni $3,16)$, in questa disponibilità a morire per gli altri si compie quanto affermato dal Vangelo: «Maiorem hac dilectionem nemo habet, ut animam suam ponat quis pro amicis suis» (Giovanni 15,13). Bernardino fornisce in questa pagina anche una descrizione ideale di come si deve assistere gli appestati: «accedit hilariter ad aegrotum, consolatur infirmum, ad patientiam exhortatur, eius necessitatibus instat, ferventer et fideliter servit, et omni debita solertia invigilat circa eum, quaerens corpori sanitatem sed magis animae sospitatem» (BERNARDINO, Quadragesimale de Evangelio Aeterno, 1956, p. 128). In filigrana si può forse intravedere qui la memoria della propria esperienza. Di sicuro, questa descrizione contiene già gli

\footnotetext{
${ }^{13}$ Bernardino presentò questo ragionamento già nel primo sermone del ciclo senese del 1425 (BERNARDINO, Siena 1425, vol. 1, p. 9). Questo passo è anche citato nel sermone de peste predicato da Bernardino da Feltre a Pavia nel 1493 (BERNARDINO DA FELTRE, Sermoni, vol. 2, 1964, p. 272).
} 
elementi che caratterizzeranno poi la descrizione dell'esemplare carità di Bernardino fatta negli articoli del processo e nella Vita di Benvoglienti.

\section{Dopo la canonizzazione: oblio e riscoperta}

Dopo la canonizzazione, l'episodio della peste sembra perdere rilevanza. Nei sermoni in onore di Bernardino è sì spesso menzionato, ma senza dargli particolare rilievo (DELCORNO, 2016, p. 237). Parallelamente, nella produzione agiografica sul nuovo santo, questo episodio viene fortemente ridimensionato. Lo si vede già nella Vita scritta da Maffeo Vegio intorno al 1455, dove il racconto serve a illustrare la «regina» delle virtù del giovane Bernardino: fortitudo e patientia. L'umanista presenta Bernardino come mosso dall'esempio della cugina Tobia («sequens vestigia Tobie consobrine») e menziona solo cursivamente la sua leadership verso i compagni (MAFFEO VEGGIO, 2014, p. 160). Un simile ridimensionamento è evidente anche nella legenda liturgica Apparuit, scritta subito dopo il testo di Maffeo Vegio e adottata per diversi anni come testo ufficiale dell'Ordine. L'Apparuit segue alla lettera Benvoglienti per la giovinezza del santo, ma con alcune omissioni significative (DELCORNO, 2016, p. 238). In particolare, parlando della peste si presenta soltanto il servizio svolto da Bernardino e la sua personale virtù. Si esclude invece ogni riferimento ai compagni e alla sua funzione di loro guida carismatica (APPARUIT, 2014, p. 301).

Considerando questa tendenza, appare sorprendente la scelta fatta nel 1481 dalla Vita scritta da Ludovico da Vicenza. Si tratta di un testo scritto da un personaggio di spicco della dirigenza osservante (CALIÒ, 2007), un testo poco studiato ma fondamentale nell'elaborazione e diffusione del culto di san Bernardino. Questa Vita era stata infatti commissionata dal capitolo generale dell'Osservanza cismontana del 1481 e ufficialmente approvata per l'ufficio della festa del santo nel capitolo del 1484, diventando così il nuovo riferimento normativo, un testo diffuso capillarmente nei breviari, anche grazie alla stampa, letto e ascoltato da tutti i frati 
ogni anno nella liturgia (SOLVI, 2009, p. 117). ${ }^{14}$ In questo modo, a 40 anni dalla morte di Bernardino, quando viene meno la testimonianza diretta dei compagni (Giacomo della Marca muore nel 1476), l'Osservanza rielabora e fissa la memoria del proprio campione di riferimento. Probabilmente la necessità di “aggiornare” l'agiografia bernardiniana col racconto della traslazione del santo nel 1472 e i relativi miracoli motivarono la richiesta di questa nuova Vita. ${ }^{15}$ In essa appaiono però molto significative anche le scelte operate nel selezionare e modulare gli episodi della vita secolare di Bernardino, i quali da soli occupano un terzo del testo. Mentre sono ridotti o esclusi diversi episodi della fanciullezza e adolescenza di Bernardino, si dà nuovamente rilevo alla sua partecipazione nella Compagnia della Vergine («locus, fons, exemplar ac schola a multis temporibus devotionis») e soprattutto si dedica grande spazio al servizio di Bernardino durante la peste (DELCORNO, 2016, p. 238). L'episodio occupa da solo ben nove lezioni nel breviario, cioè metà dello spazio dalla vita in seculo, recuperando e anzi ampliando elementi presenti in Benvoglienti e omessi da Maffeo Vegio e dall'Apparuit.

Nel racconto fatto da Ludovico, la scelta di Bernardino di offrirsi per questo servizio è presentata come frutto della meditazione assidua del detto evangelico «nessuno ha un caritas più grande di questo, dare la vita per i propri amici» (Giovanni 15,13) e della Passione di Cristo: «illud Salvatoris dictum mente revolvens: Maiorem caritatem neminem habere, quam pro amicis animam ponere, et Christum pro salute mundi in ligno crucis eam exposuisse» (LUDOVICO DA VICENZA, 1866, p. 94). Si ribadisce poi che Bernardino agì mosso dalla carità («ardentissima caritate devictus»), negando esplicitamente che la sua scelta sia stata frutto di un voto fatto chiedendo in cambio di essere salvato dal contagio («non quo pacto posset horrendam hanc pestem evadere meditare cepit, sed ...»). Di fatto, Ludovico da Vicenza sembra qui voler confutare la versione riportata dalla Vita 'Clementissimus', scritta subito dopo la canonizzazione di Bernardino. In questa vita,

\footnotetext{
${ }_{14}$ Per la collocazione nel breviario, ho tenuto conto di Breviarium, (1494, fol. 188v-184r) (ufficio per la traslazione e per la festa di san Bernardino). Il testo venne stampato anche autonomamente in latino (Vita et miracula Beati Bernardini Senensis [Vicenza: Jacobus de Dusa, c. 1482]) e volgarizzato in italiano (LEGENDA DE SANCTO BERNARDINO, 1513).

${ }^{15}$ Su mandato papale, Ludovico da Vicenza aveva presieduto il capitolo svoltosi a L'Aquila nel 1472.
} 
un atterrito Bernardino («quodam humano timore perterritus») avrebbe fatto voto di servire nell'ospedale sei mesi qualora fosse scampato alla peste («se vovit in hospitali Sancte Marie dela Scala [...] per sex menses in obsequiis se mancipare, si posset illam horridam pestem evadere illesus»). Si spostava così il servizio di Bernardino a dopo la peste, quale adempimento del voto fatto, trasformandolo in un servizio ai pellegrini e agli infermi, certo lodabile ma assai meno memorabile. ${ }^{16}$ Questa versione, seppur minoritaria, contrastava con quanto affermato nel processo di canonizzazione e doveva essere una spina nel fianco per Ludovico da Vicenza, perché questi inserisce nella propria Vita un inedito dialogo tra Bernardino e il rettore dell'ospedale. Quest'ultimo sembra quasi voler frenare il generoso slancio del giovane, dandogli così l'occasione di ribadire la sua ferma disponibilità a morire servendo gli altri sull'esempio di Cristo. ${ }^{17}$

Mentre Maffeo Vegio e l'Apparuit avevano eliminato il "sermone" di Bernardino ai compagni, Ludovico da Vicenza recupera questo elemento. In questo, si rifà anche a Giovanni da Capestrano che aveva rielaborato questo passo di Benvoglienti, assimilando ancora di più il discorso di Bernardino a un vero sermone. Capestrano infatti introduce una specie di thema biblico, là dove si dice che Bernardino esorta i compagni «allegans eis Christi verbum dicentis: Quod uni ex his minimis fratribus meis fecistis, mihi fecistis», una citazione assai appropriata tratta da Matteo 25, il giudizio finale basato sulle opere di misericordia. Inoltre, Capestrano esplicita il tema del martirio, dicendo che il giovane è «quasi martyrii praedicator effectus» ed esorta i compagni dicendo che, se moriranno, saranno considerati «tamquam martyres pro Christi nomine». Infine, pur restando centrale il tema della ricompensa eterna («Est enim Dominus fidelis remunerator»), è la parola caritas

\footnotetext{
${ }^{16}$ «Qui [Bernardinus] cum [...] pestem validam in civitate prefata vigere conspiceret, quodam humano timore perterritus, dovotione se vovit in hospitali Sancte Marie de la Scala in eadem civitate constructo per sex menses in obsequiis se mancipare, si posset illam horridam pestem evadere illesus. Et sic, Deo dante et meritis gloriose Virginis suffragantibus, illo intacto, tota illa pestis cessavit, et sicut iuvenis bone indolis et timoratus quod disposuit adimplere curavit. Et licet nobilis esset et delicatus, non horruit tamen ibi manere, ubi quasi omnes velut locum immundum perhorrescunt, sed potius se dedit in obsequiis infirmorum peregrinorum et aliarum miserabilium personarum propter amorem illius qui totus plagatus pro nobis pependit in cruce» (Vita 'Clementissimus', p. 72).

${ }_{17}$ "Cui mature e converso ardenti animo Bernardinus, pro Christo mori nequaquam timens, ait: "Potens est Deus, si voluerit, et vitam incolumem mihi servare et infirmis auxilium praestare; quod si aliud statuerit, eademque caede ac morbo me [providerit] moriturum, [equo animo] proximi gratia libens mortem suscipiam, quam Dei filius pro salute humani generis sponte suscepit" (LUDOVICO DA VICENZA, 1866, p. 94). Segno tra parentesi quadra le integrazioni derivate da Breviarium, (1494, fol. 191r).
} 
che sigilla questo breve sermone («propter Deum hoc sanctum opus egerimus charitatis») (CAPESTRANO, 1635, p. XXX).

Ludovico da Vicenza amplia ulteriormente questo passo. Infatti, in questa Vita, il "sermone" col quale Bernardino «imflammavit» i compagni sviluppa poi pienamente il tema del martirio, presentando le «rationes» per cui chi espone la propria vita servendo gli appestati sarà ricompensato con la «palma del martirio». Il discorso di Bernardino è ancora più elaborato di quelli precedenti. Accanto al versetto evangelico - già menzionato da Capestrano - «Quello che avete fatto a uno di questi, lo avete fatto a me», Bernardino aggiunge una seconda citazione biblica («il regno dei cieli patisce violenza e i violenti se ne impossessano»), esortando così i compagni a fare «violenza a se stessi» per ottenere la ricompensa eterna, la corona dei martiri. A questo punto, Ludovico da Vicenza pone sulle labbra del suo giovane Bernardino un ampio brano composto da citazioni del De laude martyrï, attribuito all'epoca a Cipriano di Cartagine, qui applicato - con uno slittamento di significato molto interessante - al quasi martirio del servizio agli appestati. Riporto il testo di Ludovico, affiancando per comodità il volgarizzamento del 1513 e indicando in corsivo le citazioni del De laude martyrii.

\begin{tabular}{|c|c|}
\hline Ludovico da Vicenza, 1866 (p. 74) & $\begin{array}{c}\text { Legenda de Sancto Bernardino } \\
1513 \text { (fol. 3rv) }\end{array}$ \\
\hline $\begin{array}{l}\text { Nec nobis deesse poterit, quod voce } \\
\text { Salvatoris proponitur: “Quod uni ex } \\
\text { minimis meis fecistis, mihi fecistis” [Mt } \\
\text { 25,40]. Ecce operum merces in medium } \\
\text { posita est, sed quo excellenti modo ad eam } \\
\text { consequendam perveniamus, Evangelicis } \\
\text { praeconibus testantibus admonemur: } \\
\text { "Regnum coelorum vim patitur et violenti } \\
\text { rapiunt illud” [Mt 11,12]. Inferamus ergo } \\
\text { violentiam nobismetipsis, nec pro }\end{array}$ & $\begin{array}{l}\text { Etiamdio farebeno [i suoi compagni] el } \\
\text { dicto del salvatore, zoè: "Quello che havete } \\
\text { fato a uno de li mei minimi, havete facto a } \\
\text { mi proprio”. Eco fratelli mei la mercede de } \\
\text { l'opera c'è posta davanti, ma aciò che cum } \\
\text { più excellente modo possiamo conseguir } \\
\text { quella, aricordiamose de quello che dice } \\
\text { l'evangelio: "El regno de li cieli patisse } \\
\text { violentia e quelli che sono violenti } \\
\text { acquistano quello". Faciamo adonche a noi } \\
\text { violentia, e non temiamo de morire per }\end{array}$ \\
\hline
\end{tabular}


proximo $^{18}$ mori pertimescamus! Quid tam magnum atque pulchrum quam pacis tempore ad martyrii coronam pervenire? Si morimur, pro Christo certe morimur, qua morte et merces augetur, et omne crimen excluditur, et omnis consummatio et status vitae martyrio decoratur. Hoc

fundamentum vitae et fidei! Hoc praesidium salutis! Hoc vinculum libertatis et honoris!19 Mors quippe integriorem facit vitam, mors magis ducit ad gloriam. ${ }^{20}$ Quid enim nobis cum hac luce, quibus lux aeterna promittitur? Quid cum hujus vitae naturaeque commercio, quos coeli altitudo deposcit? ${ }^{21}$ Intra omnium gloriam pulchrior est pro Christo mori titulus, et integrior corona signatur.

Porro contemnenda nobis mors est, quibus Christus occisus est. Ante oculos quaeso nobis concurrant Dominicae Passionis exempla, concurrant oblata praemia $^{22}$, quoniam misericordiam praestantes misericordiam consequentur. l'amore de Iesù Christo. Che cosa po esser più grande e più degna che in tempo de pace pervenire a la corona del martyrio? Se nui moriremo per l'amore de Iesù Christo veramente moriremo, per la qual morte $e l$ merito ne vien crasciudo e ogni peccato ne è perdonato: tutto el stato de la nostra vita è ornato de la palma del martyrio.

Questo è fundamento de la vita e fede nostra! Questo è a noi presidio de la salute! Questo è vinculo de linertade e honore! La morte in verità ne dà una vita più perfecta, la morte è quella che ne conduse a la gloria. Che habiamo noi a fare cum questa terrena vita, a li quali ne è promessa la glioria eterna? Che stima dovemo noi fare de questo misero mondo, con ciò sia che continuamente li excelsi cieli ce chiamano? Fra tutte quante le glorie, niuna è più degna che morir per amore de Christo, per la qual cosa, fratelli mei, la morte è da essere despreciata da noi, per li quali Christo fo crucifixo. Prego vegnavi uno poco a memoria la sua passione, vegnavi li divini premii, perché ciascaduno che miseridordia farà, misericordia senza dubio conseguirà.

\footnotetext{
${ }^{18}$ In Breviarium 1494 vi è la significativa variante: «pro Christo»

${ }^{19}$ «Considerare enim utique debetis [...] tantoque temporis tractu mundi crimen exceptum, unius ictus remediis expiare, quo et augeri merces et crimen possit excludi. Unde omnis consummatio et status vitae in martyrio est collocatus. Hoc est fundamentum vitae et fidei, hoc praesidium salutis, hoc vinculum libertatis et honoris...» (DE LAUDE MARTYRII, col. 789).

${ }^{20}$ DE LAUDE MARTYRII, col. 791.

${ }^{21}$ "Quid enim tibi cum hac luce, cui lux aeterna promissa est? Quid cum hoc vitae naturaeque commercio, quem coeli amplitudo deposcit?» (DE LAUDE MARTYRII, col. 792).

22 «Porro autem contemnenda tibi mors est, cui Christus occisus est. Ante oculos tibi, quaeso, nobis concurrant Dominicae passionis exempla, concurrant oblata et praemia ...» (DE LAUDE MARTYRII, col. 793).
} 
Segue poi la dettagliata descrizione del servizio svolto da Bernardino e dai compagni, i quali - secondo un diffuso topos della letteratura agiografica - devono anche vincere la resistenza di amici e parenti che vorrebbero trattenerli. In maniera significativa questo racconto - e non il successivo discernimento vocazionale che porterà Bernardino a entrare nei Francescani - chiude la parte dedicata alla conversatio Bernardini in seculo, sigillata dall'agiografo dicendo che «talia fuerunt in sacra aede virginis a Bernardino sanctimoniae posita firmissima fundamenta» (LUDOVICO DA VICENZA, 1866, p. 94). Il solido fondamento della santità di Bernardino è rappresentato appunto dalla sua caritas cristomimetica e dal servizio svolto come secolare nell'ospedale senese.

In maniera sorprendente, quello della peste è l'episodio più lungo e dettagliato di tutta questa legenda. Inoltre, l'unico sermone di Bernardino inserito in questa Vita è quello rivolto ai suoi compagni. Dopo ci si soffermerà sul suo ministero di predicatore, ma senza più riportare le sue parole. Come mai? Perché quest'episodio ha tutta questa rilevanza? Mentre nella vita scritta da Benvoglienti era chiara l’intenzione di rafforzare il legame tra la fase senese della vita di Bernardino e la sua santità, più difficile è individuare le ragioni che muovono Ludovico da Vicenza, considerando che - è bene ribadirlo - egli scrive un testo per la liturgia dell'Ordine, destinato cioè a plasmare - almeno nelle intenzioni - la memoria del santo e l'autocomprensione stessa dell'Osservanza. ${ }^{23}$

L'episodio serviva sicuramente a mettere in luce la disponibilità di Bernardino al martirio, inserendolo così in una consolidata tradizione di santi francescani, da Francesco ad Antonio, e agli stessi martiri del Marocco, il cui culto pubblico era stato approvato proprio nel 1481 . Vi poteva tuttavia essere un'ulteriore ragione per insistere su questo episodio. La raffigurazione di Bernardino che, con l'esempio e la parola, conduce la città fuori da un'emergenza insieme sanitaria e spirituale - ovvero intrinsecamente politica - proponeva un modello di santità funzionale all'evoluzione

${ }^{23}$ Si vedano le riflessioni sviluppate riguardo all'ufficio liturgico per san Francesco in Johnson (2011). 
dell'Osservanza minoritica e al suo legame sempre più stretto con la vita delle città italiane. In questo episodio, Bernardino è presentato come un santo nel cuore della città, come capace di rispondere a un'emergenza, radunando altre persone e convincendole ad anteporre i bisogni collettivi ai propri, anche a rischio della propria vita. Il racconto tracciava così un possibile paradigma per la leadership dei frati dell'Osservanza all'interno delle città e ribadiva la necessità per i cives di anteporre il bonum commune al bonum proprium, ovvero l'architrave di un intero progetto di società, divulgato in quegli anni dalla predicazione osservante. Del resto, nei sermoni di Bernardino e dei suoi discepoli, la peste diventava facilmente simbolo - quando non visibile conseguenza - delle infermità della società (parzialità, sodomia, usura) verso le quali si indirizza l'intervento della predicazione osservante, idealmente tesa a curare e purificare la città. ${ }^{24}$ Lo mostra chiaramente un passo dello stesso Bernardino. Predicando a Siena nel 1427, «attraverso la concretezza della sua acuta memoria olfattiva» (PICCINNI, 2003, p. 44), egli ricorda esplicitamente la pestilenza del 1400, trasformandola in metafora della sodomia:

Doh, ode: sai come fa questo peccato? Fa come fa la pistolenzia. Ècci chi si ricordi de la mortalità del quatrocento? Elli era tanta puzza a lo Spedale, che non vi si poteva abitare solo per la puzza de la pistolenza. El riparo era questo, che vi si faceva molto fummo e fuoco: questo era il migliore riparo che vi si potesse fare. Così si vorrebbe fare a volere purgare questo peccato: fuoco e fumo: parole di lusinghe e promesse, parole di predicazione, che ora mentre ch'io predico di questo peccato, discenda il fuoco da cielo e purga le menti di voi che mi state a udire (BERNARDINO, Siena 1427, p. 1152). ${ }^{25}$

Se durante la peste si cercava di difendersi dal morbo purificando l'aria con fumigagioni, nel suo ministero Bernardino continua quel "servizio" ma su un altro

\footnotetext{
24 il già citato sermone de peste di Bernardino da Feltre è molto esplicito in questo: «omnia flagella et pestes veniunt a Deo». La cuasa sono i peccati degli uomini e l'assenza di carità: «propter blasfemias et peccata contro natura veniunt pestes [...]. Tu vis pur opprimere pauperes, et Deus mittet pestem. [...] Usurarii et fautores, receptores judeorum, fano venir pestem [...] Propter hoc sepe deus mittit pestem, per la pocha charità» (BERNARDINO DA FELTRE, Sermoni, vol. 2, 1964, p. 269-271). Ulteriori esempi in Hanska (2002, p. 116-143), compreso un interessante sermone di Bernardino de Bustis.

${ }^{25}$ Questo dettaglio è ripetuto in molte delle descrizioni della peste, a partire da Benvoglienti che, forse, lo attinge da questo passaggio dei sermoni: «Crebros parabat ignes et fumigia odorifera, acetaque multa spargebat fere per universam domum, ut fetor et corruptus aer pro parte saltem rectificaretur et libentius a ministris provideretur infirmis» (BENVOGLIENTI, 1902, p. 70).
} 
piano: la sua predicazione deve essere come un fuoco che purifica le menti dalla pestilenza della sodomia e degli altri vizi che ammorbano la città.

\section{Gli affreschi di Lodi}

Se l'Osservanza minoritica poteva scorgere in questo episodio un modello della propria azione pastorale e del proprio protagonismo nella vita delle città, il tema del servizio di Bernardino durante la peste poteva essere utilizzato anche in specifici contesti cittadini, come mostra il caso di Lodi, in Lombardia. In questa città, infatti, pochi anni prima della vita scritta da Ludovico da Vicenza, un'eccezionale agiografia dipinta dedicò grande attenzione al servizio di Bernardino presso l'Ospedale della Scala. Si tratta del ciclo di affreschi nella cappella dedicata a questo santo nella chiesa di San Francesco, dipinto nel 1476-1477 da Gian Giacomo da Lodi (FARAONI, 2011, p. 142-146 et BANDERA, 1998). Ventitré scene, accompagnate da didascalie latine non sempre pienamente leggibili, visualizzano la vita del santo senese su due intere pareti. ${ }^{26} \mathrm{Si}$ tratta del più ampio ciclo pittorico con scene della vita di san Bernardino in tutto il Quattrocento e l'unico che racconti nel dettaglio la sua vita prima della vestizione religiosa (BISOGNI, 1982, p. 390). ${ }^{27}$ Su una parete è raffigurata la vita in seculo (fig. 3), suddivisa in tre sezioni: infanzia, adolescenza e giovinezza (DELCORNO, 2016). Sull'altra parete è narrata la sua vita religiosa, dall'ingresso nell'Ordine alla morte, e alla traslazione del 1472 (fig. 4). Tutto il ciclo lodigiano meriterebbe un approfondito studio complessivo. Un dato però emerge subito in tutta evidenza: il sorprendente rilievo che assume l'episodio del servizio di Bernardino presso l'Ospedale della Scala, il quale occupa quasi un terzo dello spazio, con ben otto scene.

\footnotetext{
${ }^{26}$ Un'ipotetica ricostruzione delle didascalie in BIAGINI, 1896, p. 116-124.

${ }_{27}$ Un'eccezione parziale sono le scene della preghiera a Porta Camollia e del giovane Bernardino eremita penitente, affrescate da Pinturicchio nella Cappella Bufalini dell'Aracoeli a Roma.
} 
Nella prima scena Bernardino si offre volontario e riceve dal rettore le chiavi dell'ospedale (fig. 5). ${ }^{28} \mathrm{Al}$ centro si mostra Bernardino che, presentando le necessità dei poveri, convince $i$ «consocios valde delicatos» a porsi al loro servizio (fig. 6). ${ }^{29} \mathrm{Le}$ due scene successive lo mostrano mentre accoglie i pauperes e lava loro i piedi (fig. 7), riprendendo volutamente l'iconografia della lavanda dei piedi di Gesù. ${ }^{0}$ Nella parte inferiore dell'affresco, Bernardino, assistito da un compagno, medica le ferite di un malato (fig. 2). Egli è poi ritratto prima in cucina, chino davanti al focolare (fig. 8), e poi mentre insieme a un compagno serve i malati a tavola (fig. 9). Infine, nell'ultima scena, Bernardino seppellisce un morto nella cappella dell'ospedale (fig. 10). Pur senza seguire strettamente - almeno guardando alle didascalie - una delle Vite, è chiaro che gli affreschi attingono alla vivida descrizione già contenuta in nuce nell'articolo del processo (vedi sopra) e delineata con abbondanza di dettagli nella Vita di Benvoglienti, con la quale queste pitture condividono anche il rilievo dato agli altri protagonisti della vita della città, quali i compagni e i reggenti dell'ospedale.

In questo modo, gli affreschi di Lodi proponevano l'esemplare carità di Bernardino come modello adatto anche - e soprattutto - per i laici, mostrando un esempio imitabile, tanto più vicino perché recente (MATTIOLI ROSSI, 1983, p. 236). ${ }^{31}$ L'accento non cade, come in altri cicli pittorici, sui miracoli operati da Bernardino, ma domina «un atteggiamento assai concreto e didattico» intento a raffigurare, in particolare nella giovinezza di Bernardino, "un esempio di apprendistato alla virtù e alla santità» (BISOGNI, 1982, p. 390). Si mostra così il santo quale civis esemplare che non solo compie le opere di misericordia, ma è capace di ricomporre il tessuto sociale della città, unendo intorno a sé le autorità cittadine, i nobili compagni e i poveri, facendosi in un certo senso promotore di un

\footnotetext{
${ }^{28}$ Didascalia: «Qualiter civitate Senarum infecta peste et existentibus pauperibus hospitalis sine regimine, obtulit se paratum deservire et ipsi claves presentaverunt gubernatores dicti hospitalis».

${ }^{29}$ Didascalia: «Qualiter multiplicatis tot pauperibus non poterat solus supplere et adhibuit secum alios servientes consocios suos valde delicatos».

${ }^{30}$ Le due scene sono unificate da un'unica didascalia: «Qualiter propriis manibus pauperes hospitale conducebat eis pedes abluendo». Esplicita questo tema l'Apparuit, dicendo che Bernardino «iuxta dominicum preceptum curvatis genibus abluebat pedes eorum» (APPARUIT,2014, p. 301).

${ }^{31}$ Sul rapporto tra santità ammirabile e santità imitabile, Vauchez (1991).
} 
nuovo patto, basato sulla caritas. Lo si vede in particolare nella scena posta al centro della parete, dove egli svolge un ruolo di mediatore (fig. 6).

L'interesse per l'episodio della peste era - come si è visto - diffuso nelle vitae. Sorprende però lo spazio dedicatovi in questi affreschi. Se ci si poteva forse aspettare questa peculiare raffigurazione a Siena, perché invece venne fatta a Lodi? I dati sulla committenza degli affreschi non aiutano - almeno allo stato attuale della ricerca - a individuare le ragioni di questa scelta. Gli affreschi vennero pagati grazie al testamento del patrizio Luigi Bononi, dettato il 2 gennaio 1476, e sul muro della cappella, una scritta - ora scomparsa - recitava: «1477 die 8 novembris dominus Aloysius Bononus fecit fieri» (FARAONI, 2011, p. 142). Vista la collocazione degli affreschi, al di là del ruolo della famiglia Bononi, nell'elaborazione del progetto iconografico si deve pensare anche a una partecipazione attiva dei minori conventuali, di cui erano la chiesa e l'annesso convento. $3^{2}$ Il fatto che il più ampio ciclo pittorico sulla vita di Bernardino si trovi in una chiesa conventuale rompe certi schemi storiografici ("Bernardino, il santo dell’Osservanza”) e permette di vedere come i vari rami della litigiosa famiglia minoritica guardassero a questo santo con diverse e parallele strategie di appropriazione. Questo però non ci fornisce ancora chiarimenti sulla scelta di concentrarsi sull'episodio della peste.

Alcuni indizi significativi sui motivi di tale scelta emergono invece guardando al contesto sociale, politico e religioso di Lodi. In quegli anni, la città stava infatti sperimentando una radicale riforma del sistema ospedaliero, seguendo in questo l'esempio di altre città lombarde (ALBINI, 2000).33 Se già nel 1454 il consiglio cittadino aveva istituito una commissione di riforma, bisognò attendere il 1457 e l'inizio dell'episcopato di Carlo Pallavicino perché venisse approvata la costruzione di un grande ospedale cittadino, iniziata poi effettivamente nel 1459 (GORINI, 1998 et ALBINI, 1993, p. 109 e 115). In nome di una maggiore efficienza e di una medicalizzazione dell'assistenza, i precedenti ospedali di fondazione medievale

\footnotetext{
${ }^{32}$ La chiesa e il convento passarono agli Osservanti nel 1527 (Granata, 1983).

${ }_{33}$ Per un quadro ampio e aggiornato sulla riforma del sistema ospedaliero, Bianchi \& Słoń (2006) e Frank (2014).
} 
venivano soppressi, accentrando il loro patrimonio e la loro funzione in una nuova istituzione, fondata presso l'antico Ospedale di Santo Spirito della Caritade. Tuttavia, le tensioni tra il nuovo ospedale, che iniziò a operare nel 1467, e gli antichi ospizi, che non volevano chiudere, continuarono per decenni (CREMASCOLI \& DONINI, 1998, p. 9-36). Se si conta inoltre che i lavori del nuovo ospedale continuarono per tutto il Quattrocento (GORINI, 1996), si può ragionevolmente considerare che gli affreschi sulla vita di san Bernardino vennero dipinti quando il tema della riforma del sistema ospedaliero era ancora assai rilevante nel dibattito cittadino.

In questo contesto, è possibile individuare dei collegamenti tra gli affreschi e il nuovo ospedale. Anzitutto l'Ospedale de la Caritade si trovava accanto alla piazza dove si affaccia la chiesa di San Francesco. Quanti vedevano il servizio di Bernardino ampiamente illustrato in questi affreschi potevano facilmente collegarli all'ospedale posto a pochi metri da quella chiesa. Un collegamento ancor più significativo lo si trova nel decreto con cui il vescovo concedeva l'accentramento degli antichi ospedali (21 novembre 1457), dove si afferma che il nuovo ospedale avrebbe seguito il modello di quelli di Firenze e, appunto, Siena: «ad instantiam Florentini et Senensis hospitalium» (ALBINI, 1993, p. 115).34 L'Ospedale della Scala era il modello principale di sistema assistenziale accentrato a cui si ispiravano i nuovi ospedali lombardi, là dove il sistema fiorentino prevedeva invece l'autonomia delle singole strutture, potenziate attraverso una più accentuata divisione dei compiti, pur appartenendo entrambi i modelli «a un unico e ampio progetto di riforma» (BIANCHI \& SŁOŃ, 2006, p. 20-21). Nel 1401, Gian Galeazzo Visconti, duca di Milano, decretò di costruire un nuovo ospedale cittadino (fondato poi solo nel 1456) «modo et forma quibus regulatur et gubernatur hospitale civitatis Senarum». A tale proposito, egli aveva chiesto (probabilmente già nel 1399) un resoconto sull'organizzazione dell'assistenza e la forma architettonica del famoso ospedale senese, fotografandone così la situazione all'epoca della peste del 1400

\footnotetext{
${ }^{34}$ Nelle copie del documento c'è un'oscillazione tra senensis (prevalente) e venetorum, con un probabile rimando al modello di Brescia, basato a sua volta su quello senese (vedi nota 36) (CREMASCOLI \& DONINI, 1998, p. 19).
} 
(LEVEROTTI, 1981).35 Nel 1427 il consiglio cittadino di Brescia (passata di recente sotto il controllo di Venezia) decretava: «hospitale unum magnum et generale fiat Brixie sicut in civitate Senarum»(GATTI PERER \& MAGGIONI, 1989, p. 153).36 Similmente l'ospedale generale di Pavia, fondato nel 1449, è definito «ad instar Florentinensis et Senensis hospitalium» nella bolla di approvazione di Niccolò V, e uguali riferimenti al modello senese si trovano nei documenti relativi ai nuovi ospedali di Cremona e Milano (PICCINNI, 2003, p. 20-21).

Ecco allora che gli affreschi di San Francesco di Lodi davano indubbiamente grande visibilità proprio a questo prestigioso modello, l'Ospedale della Scala di Siena, mostrandone non tanto la realtà concreta, ma il suo perfetto funzionamento sotto la guida di Bernardino. Tutto questo poteva rappresentare un prezioso supporto nel creare consenso intorno a un'analoga istituzione, nuova e ancora in parte avversata. Gli affreschi della cappella di San Bernardino fornivano un'indiretta "pubblicità" o propaganda a favore della nuova istituzione cittadina, veicolando il messaggio che, come a Siena il famoso Ospedale della Scala promuoveva la salute e la santità della città, un'analoga istituzione poteva svolgere lo stesso ruolo a Lodi. Si è parlato di una vera e propria «battaglia di parole» a sostegno o contro i progetti di riforma degli ospedali in età rinascimentale, cogliendo in questo l'importanza essenziale degli strumenti per costruire il consenso (FRANK, 2014). Come è noto, per promuovere i nuovi ospedali generali, in molte città (Milano, Pavia, Piacenza, Parma) le autorità civili si avvalsero del supporto della predicazione osservante: la parola del predicatore serviva a convincere i cittadini, a raccogliere fondi, ad ammantare di sacralità il nuovo progetto sociale e - non ultimo - a piegare le resistenze al mutamento.37 Per quanto riguarda Lodi non si ha notizia di un impegno diretto dei maggiori predicatori del tempo, a meno di speculare sulla presenza di Michele Carcano, uno specialista in materia, nella Quaresima del 1484 (RUSCONI,

\footnotetext{
${ }^{35}$ Sulle vicende dell'ospedale milanese, si veda ora Frank (2014, pp. 75-142).

${ }^{36}$ L'Ospedale di San Luca venne costruito solo tra 1447-1452, sui modelli senesi e fiorentini, divenendo un importante riferimento per i successivi ospedali lombardi (BIANCHI \& SŁOŃ, 2006, p. 23).

${ }^{37} \mathrm{Si}$ veda da ultimo ALBINI, 2013. Interessante in particolare l'esempio di Parma dove nelle discussioni del consiglio cittadino, nel $1489 \mathrm{ci}$ cita come auctoritas quanto affermato in precedenza da Bernardino da Feltre.
} 
1976). Invece di cercare aiuto nella viva voce del predicatore, a Lodi la promozione della riforma ospedaliera era sostenuta in un certo senso attraverso la vita esemplare di san Bernardino, dipinta- quasi come un sermone a colori - nella più importante chiesa nei pressi del nuovo ospedale.

Inoltre, se questo messaggio era rivolto a tutti gli abitanti di Lodi, in questi affreschi i membri della confraternita chiamata a reggere l'ospedale (esponenti del patriziato urbano, nominati a vita) potevano trovare un messaggio più specifico, scorgendovi una raffigurazione ideale del loro servizio e della loro spiritualità. Gli statuti di questa confraternita, scritti nel 1466, tratteggiano una spiritualità basata sull'amore dei poveri in un contesto di preghiera e devozione. In particolare, il prologo sottolinea il ruolo civile - oltre che religioso - dell'ospedale e raccomanda ai membri della confraternita di attirare con il loro esempio luminoso altre persone a una vita di carità per il bene della città:

Estote igitur lumen vobis primum et dehinc universae civitati speculum, lucerna pietatisque et sanctitatis singulare exemplum ac vestra opera nullus non admiretur et ex vobis rectam vivendi rationem quisque perdiscat ac si qui forte erunt [...] ad pietatem minus propensi vestro exemplo excitentur, rescipiscant et sese ad meliorem frugem recipiant (CREMASCOLI \& DONINI, 1998, p. 50). $3^{38}$

Di per sé, queste erano coordinate comuni a molte delle confraternite collegate a un ospedale. Tuttavia, quanti consideravano l'ospedale di Siena un modello ispiratore e i membri di una confraternita che voleva essere un esempio per la città, questi potevano guardare con particolare interesse alla dettagliata raffigurazione del servizio di san Bernardino - e dei suoi nobili compagni - nel famoso Ospedale della Scala. Di fatto, questi affreschi descrivevano un ideale religioso e civile in cui essi potevano pienamente identificarsi. Gli affreschi sulla giovinezza di Bernardino presentavano così un modello di santità fermamente connesso con la vita della città. La sua esemplare carità risultava inscindibile da

\footnotetext{
${ }^{38}$ Un quadro generale in Frank (2009) dove si considerano con attenzione anche questa confraternita e i suoi statuti, così come in Frank (2014, pp. 122-125).
} 
un'istituzione civica come l'Ospedale della Scala, una rappresentazione che assumeva un valore nuovo nel contesto locale di Lodi, presentando la carità operosa di Bernardino non solo come virtù cristiana ma anche come esempio civile.

Fig. 03: La vita in seculo di Barnardino. Gian Giacomo da Lodi. Vita di San Bernardino (1476-1477). Chiesa di San Francesco, Cappella di San Bernardino. Copyright: Pietro Delcorno.

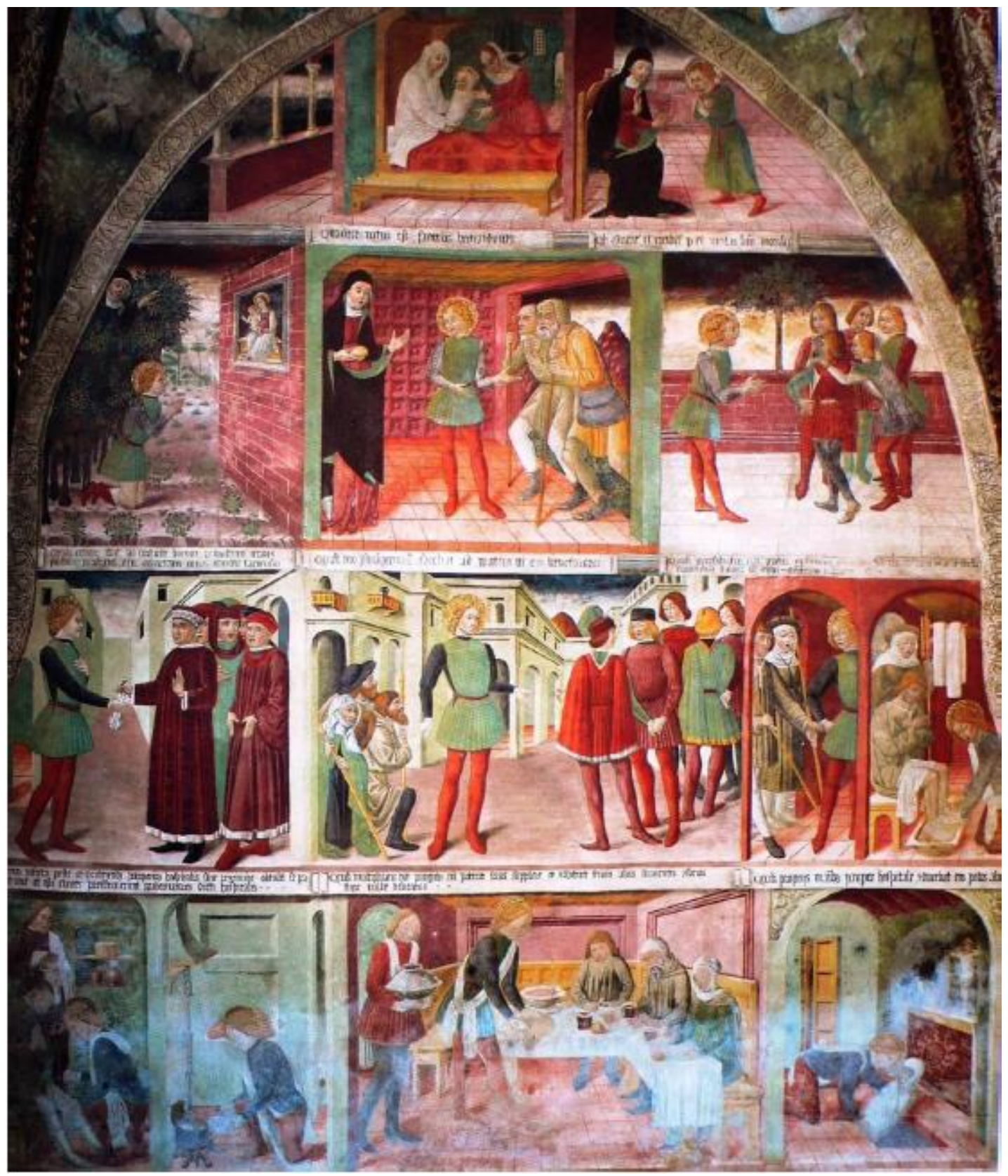


Fig. 04: La vita in religione di Bernardino. Gian Giacomo da Lodi. Vita di San Bernardino (1476-1477). Chiesa di San Francesco, Cappella di San Bernardino. Copyright: Pietro Delcorno.

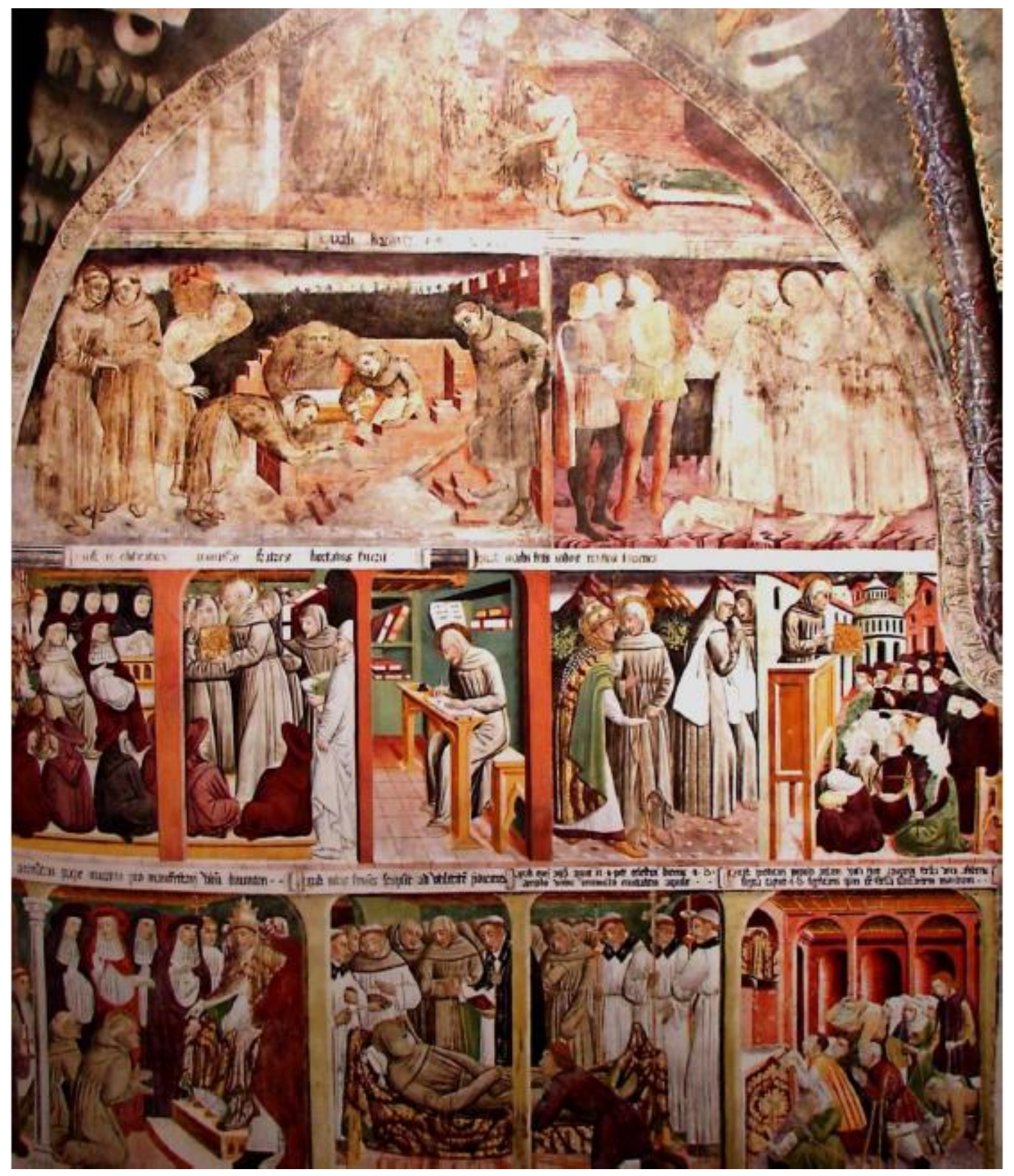


Fig. 05: Bernardino riceve la chiavi dell'Ospedale di Santa Maria della Scala. Gian Giacomo da Lodi. Vita di San Bernardino (1476-1477). Chiesa di San Francesco, Cappella di San Bernardino.

Copyright: Pietro Delcorno.

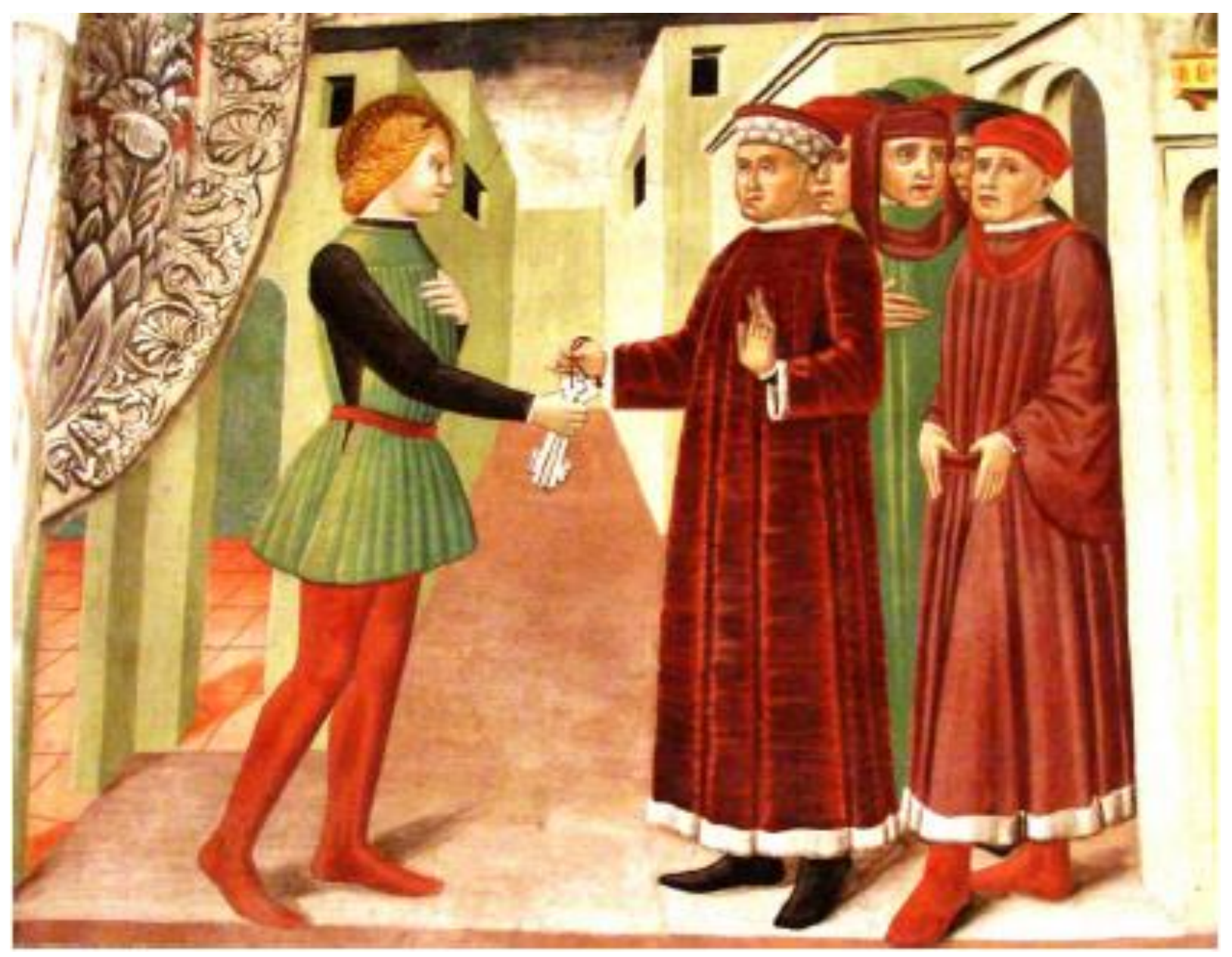


Fig. o6: Bernardino esorta i compagni a mettersi a servizio dei pauperes. Gian Giacomo da Lodi. Vita di San Bernardino (1476-1477). Chiesa di San Francesco, Cappella di San Bernardino.

Copyright: Pietro Delcorno.

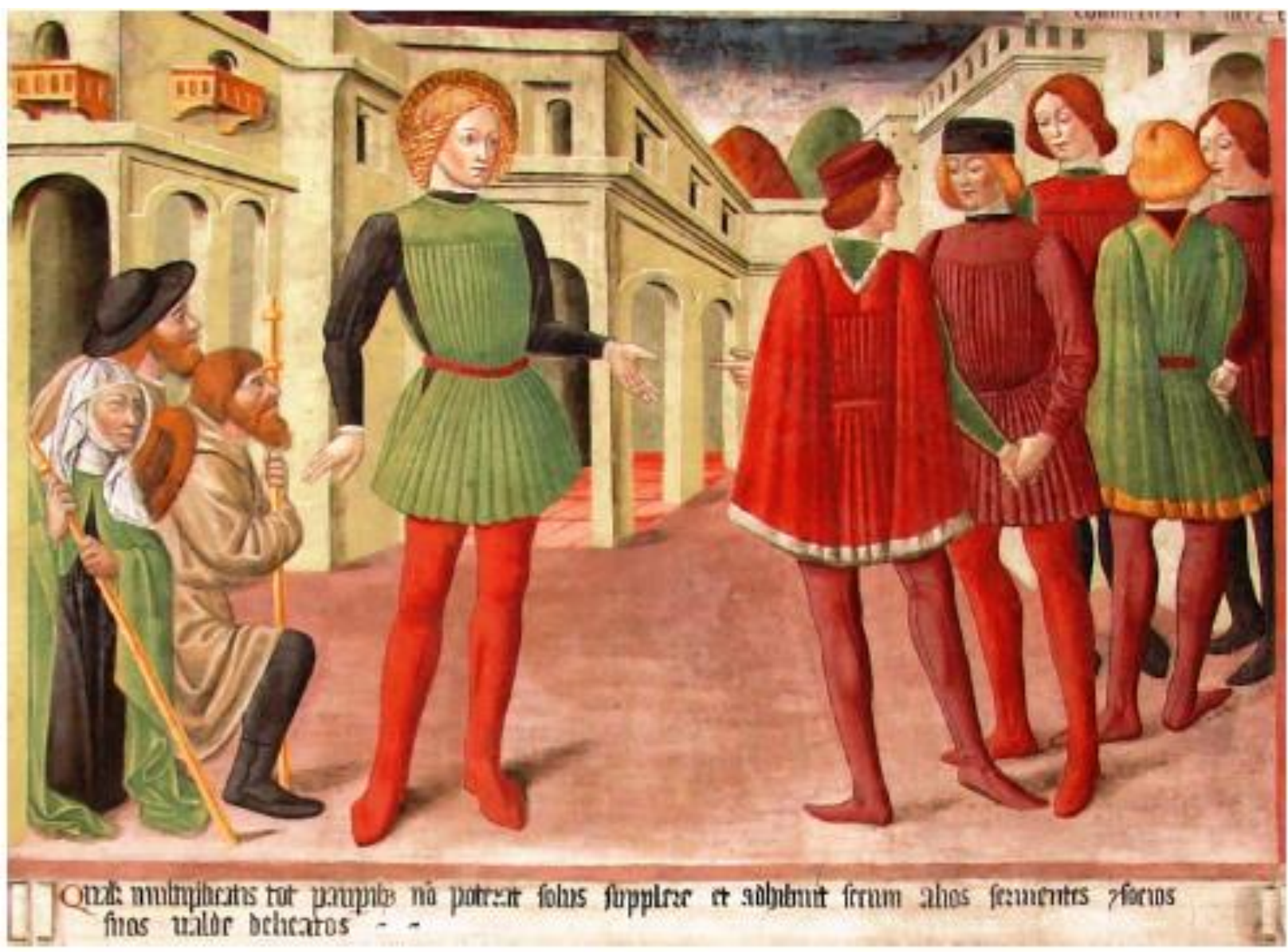


Fig. 07: Bernardino accoglie i poveri e lava loro i piedi. Gian Giacomo da Lodi. Vita di San Bernardino (1476-1477). Chiesa di San Francesco, Cappella di San

\section{Bernardino.}

Copyright: Pietro Delcorno

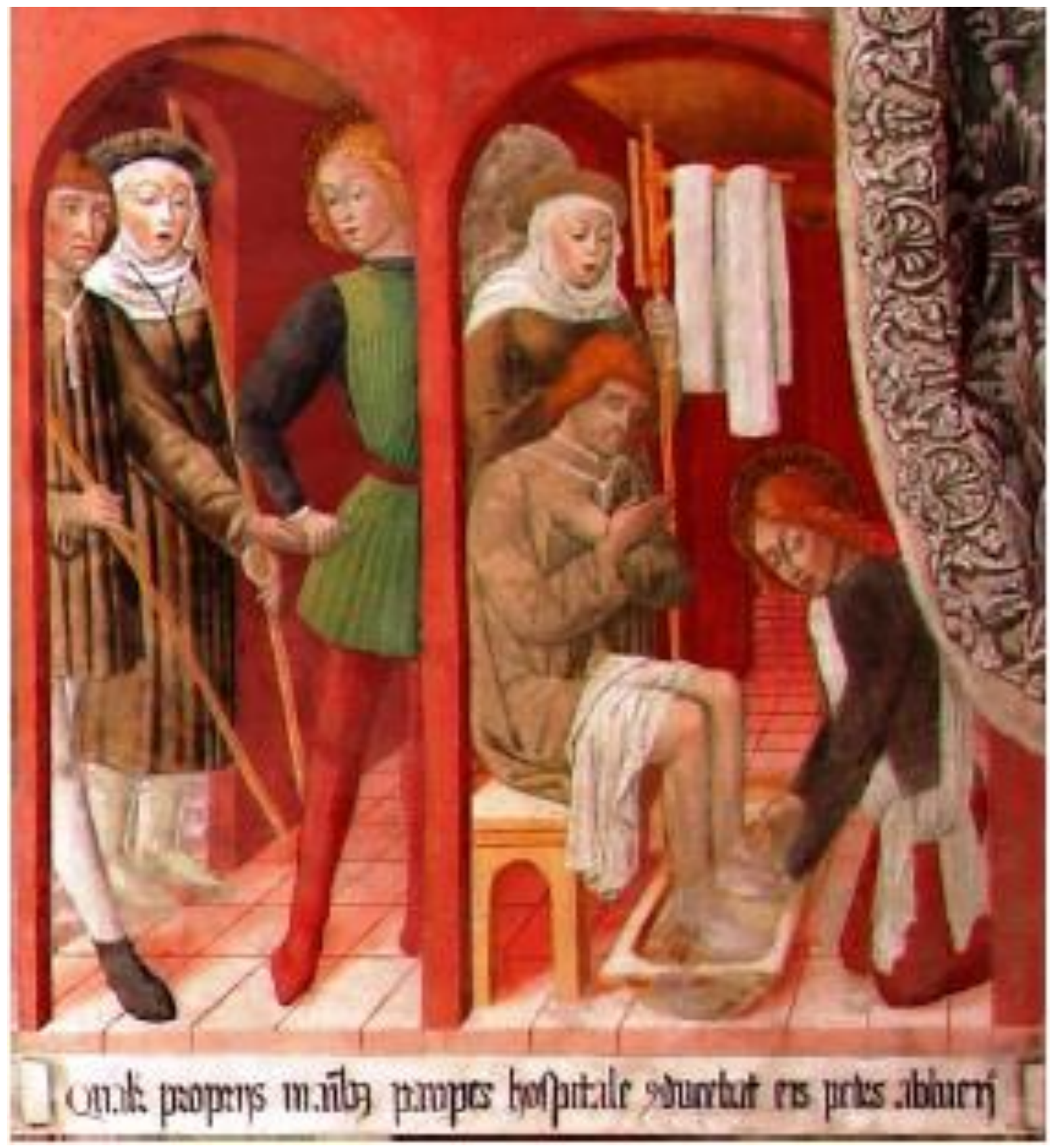


Fig. 08 : Bernardino in cucina. Gian Giacomo da Lodi. Vita di San Bernardino (1476-1477). Chiesa di San Francesco, Cappella di San Bernardino.

Copyright: Pietro Delcorno.

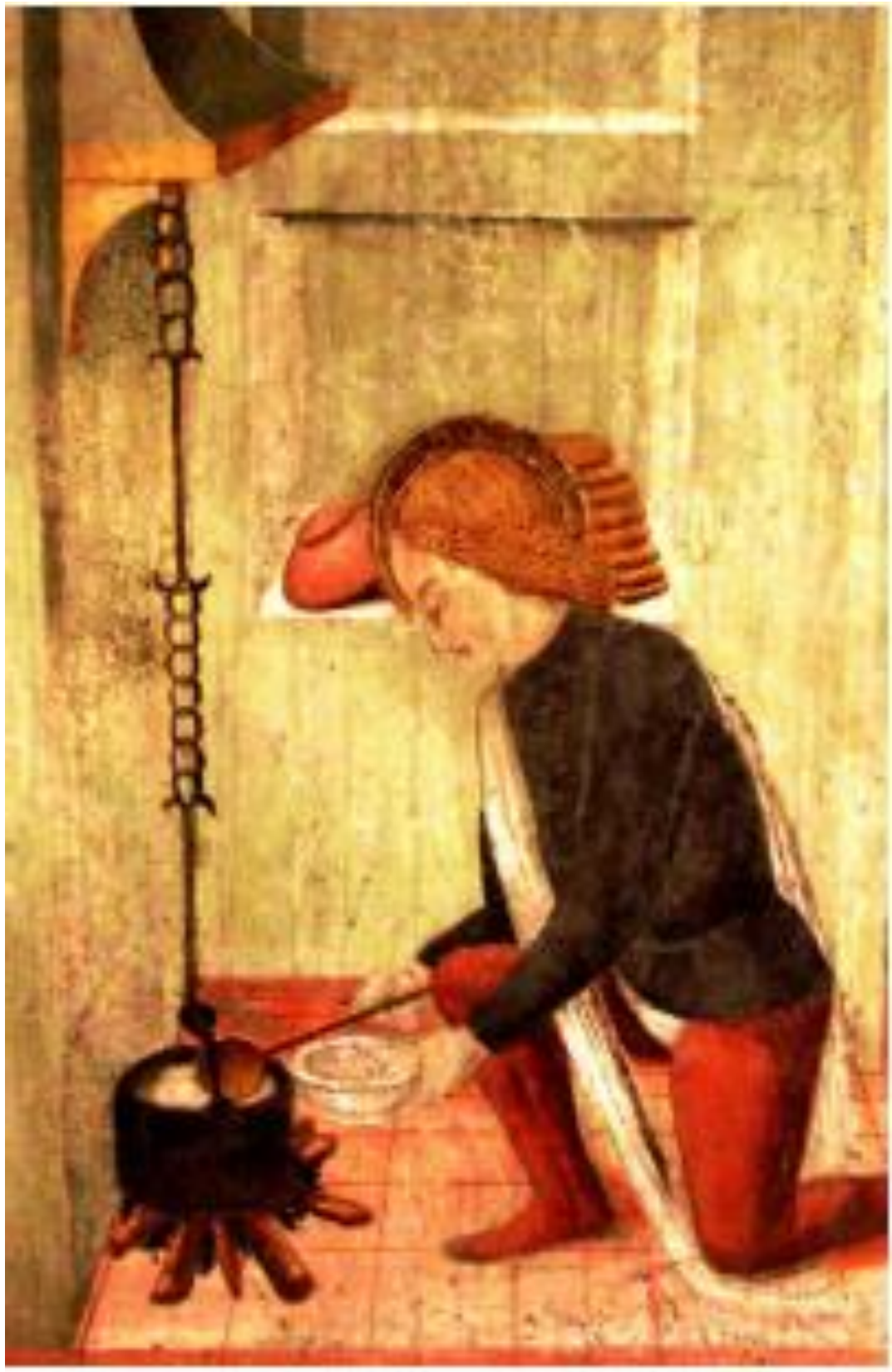


Fig. 09 : Bernardino e un compagno servono a tavola. Gian Giacomo da Lodi. Vita di San Bernardino (1476-1477). Chiesa di San Francesco, Cappella di San Bernardino.

Copyright: Pietro Delcorno.

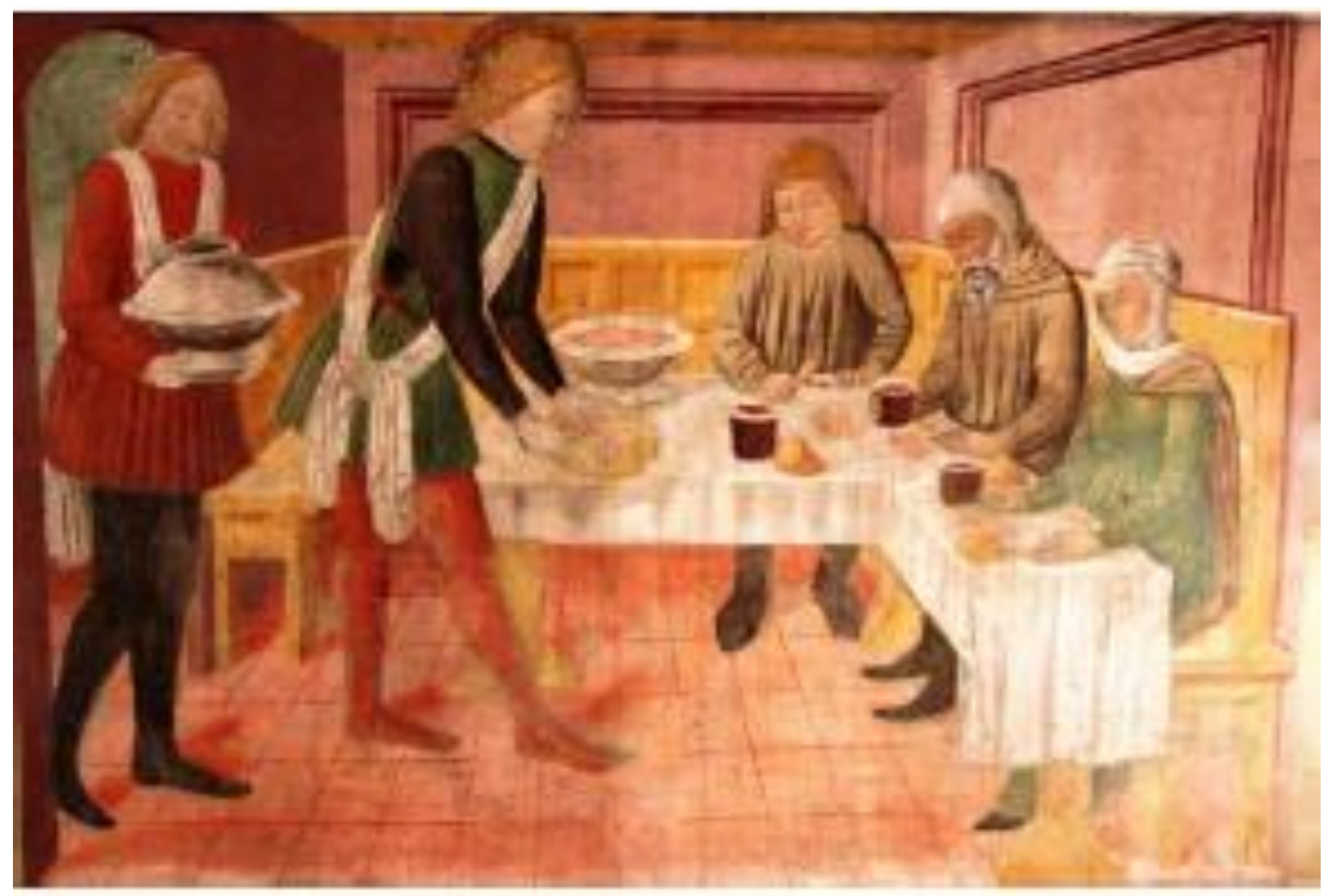


Fig. 10: Bernardino seppellisce un morto nella cappella dell'ospedale. Gian Giacomo da Lodi. Vita di San Bernardino (1476-1477). Chiesa di San Francesco, Cappella di San Bernardino.

Copyright: Pietro Delcorno.

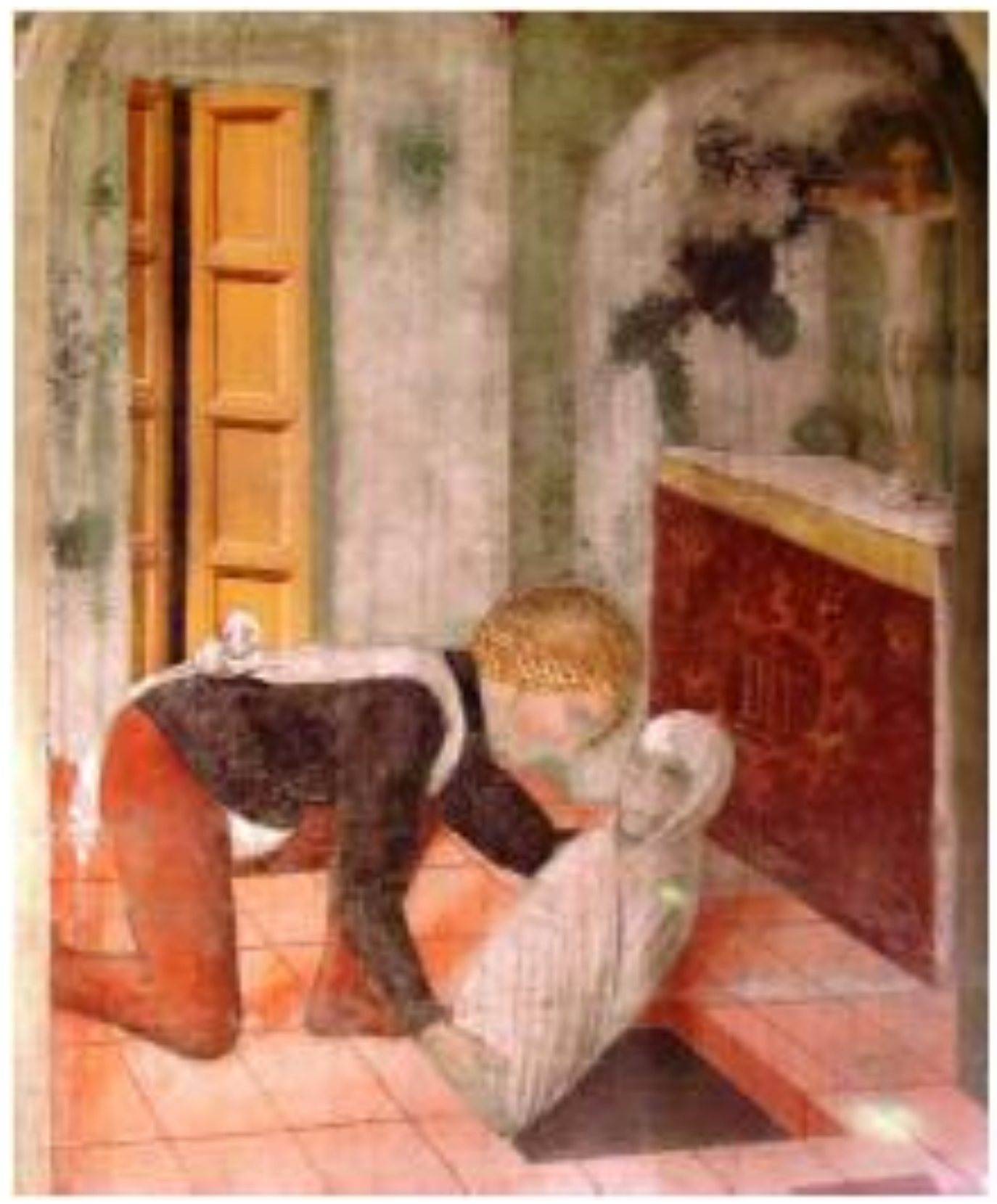




\section{Conclusione}

L'ultimo caso analizzato rende evidente - anche con la forza delle immagini come l'episodio agiografico del servizio di Bernardino durante la peste potesse servire quale strumento efficace per presentare un ideale religioso e civile che interagendo con le specifiche e mutevoli vicende dell'Ordine e delle città - assumeva una molteplice valenza politica. A Siena questo episodio - attraverso la Vita di Benvoglienti - era servito a consolidare il legame tra il santo e la città, facendo perno su una consolidata istituzione civica quale l'Ospedale della Scala, oggetto in quegli anni di un ampio programma di lavori per farne ancora di più lo specchio della carità pubblica senese. Scrivendo su incarico dell'Osservanza minoritica, Ludovico da Vicenza si era servito di questo racconto per ritrarre Bernardino come un martire della carità cristoforme e come modello di leader capace di operare nel cuore della città in vista del bene comune, fornendo così un possibile paradigma all'azione dei frati all'interno della società. Nel contesto di Lodi, l'inscindibile legame tra Bernardino e un'istituzione come l'Ospedale della Scala assumeva un valore nuovo, mostrando la carità esemplare di Bernardino non solo come virtù religiosa, ma anche come valore civile, legittimando - almeno di riflesso - l'importante riforma ospedaliera in corso. Nelle mani di abili professionisti della comunicazione religiosa e politica, la memoria agiografica dell'esemplare carità operosa del "laico" Bernardino si presentava quale strumento prezioso e flessibile, utile a veicolare diversi messaggi, «spiritualmente efficaci e politicamente influenti» (MIATELLO, 2013, p. 13), prolungando e attualizzando l'insegnamento di Bernardino «circa al ben vivere de la vostra città» (BERNARDINO, Siena, 1427, p. 1379). 


\section{RIFERIMENTI BIBLIOGRAFICI}

ALBINI, Giuliana. Città e ospedali nella Lombardia medievale. Bologna: Clueb, 1993.

ALBINI, Giuliana. L’Osservanza francescana e la pratica della carità. Uno sguardo alle città dell'Italia centro-settentrionale. In: I frati osservanti e la società in Italia nel secolo XV, Spoleto: Centro Italiano di studi sull'Alto Medioevo, 2013, p. 227-251.

ALBINI, Giuliana. La riforma quattrocentesca degli ospedali nel ducato di Milano, tra poteri laici ed ecclesiastici. In: ZAMAGNI, Vera (dir.). Povertà e innovazioni

istituzionali in Italia: Dal medioevo ad oggi. Bologna: Il Mulino, 2000, p. 95-110.

APPARUIT = Legenda 'Apparuit'. In SOLVI, 2014, p. 291-348.

BANDERA, Sandrina. Gian Giacomo da Lodi: Decorazione della cappella di San Bernardino. In: MARUBBI, 1998, p. 237-238.

BARTOLOMEI ROMAGNOLI, Alessandra. L’immagine di Bernardino da Siena nella predicazione osservante. In: SERPICO, 2009, p. 1-21.

BARTOLOMEI ROMAGNOLI, Alessandra. Osservanza francescana e disciplina del culto dei santi. Modelli di perfezione e strategie di riforma nell'opera di Giovanni da Capestrano. In: PÁSZTOR, Edith (dir.). Ideali di perfezione ed esperienze di riforma in $\mathbf{S}$.

Giovanni da Capestrano (Capestrano, 1-2 dicembre 2001). Capestrano: 2002, p. 127153.

BENVOGLIENTI, Leonardo. Vita. In: VAN ORTROY, François. Vie de S. Bernardin de Sienne par Léonard Benvoglienti. In: Analecta Bollandiana, ${ }^{\circ}$ 21, 1902, p. 53-80.

BERNARDINO DA FELTRE, Sermoni. In: VARISCHI, Carlo (ed.). Sermoni del beato Bernardino Tomitano da Feltre. Milano: Cariplo, 1964.

BERNARDINO DA SIENA. Quadragesimale de Evangelio Aeterno. In: BERNARDINO DA SIENA, Opera omnia [...] studio et cura PP. Collegi S. Bonaventurae. Quaracchi: Typographia Collegii S. Bonaventurae, 1950-1965, vol. 3-5 (1956).

BERNARDINO, Siena 1425 = BERNARDINO DA SIENA, Le prediche volgari. Predicazione del 1425 in Siena, a cura CANNAROZZI, Ciro. Firenze: Rinaldi, 1958.

BERNARDINO, Siena 1427 = BERNARDINO DA SIENA, Prediche volgari sul Campo di Siena 1427, a cura di DELCORNO, Carlo. Milano: Rusconi, 1989.

BIAGINI, Enrico. Monografia storico-artistica della Chiesa di S. Francesco in Lodi - Parte seconda. In: Archivio Storico di Lodi, s. II, nº 15/3, 1896, p. 97-129. 
BIANCHI, Francesco \& SŁOŃ, Marek. Le riforme ospedaliere del Quattrocento in Italia e nell'Europa centrale. In: Ricerche di storia sociale e religiosa, $n^{\circ} 35 / 69,2006$, p. 745 .

BISOGNI, Fabio. Per un census delle rappresentazioni di S. Bernardino da Siena nella pittura in Lombardia, Piemonte e Liguria fino agli inizi del Cinquecento. In: MAFFEI, Domenico (dir.) \& NARDI, Paolo (dir.). Atti del simposio internazionale cateriniano-bernardiniano (Siena, 17-20 aprile 1980). Siena: Accademia degli Intronati, 1982, p. 373-392.

BREVIARIUM ROMANUM. Proprium de sanctis. Venezia: Andrea Torresano, 1494.

CACIOTTI, Alvaro (dir.) \& MELLI, Maria (dir.). Giovanni da Capestrano e la riforma della Chiesa. Milano: EBF, 2008.

CALIÒ, Tommaso. Ludovico da Vicenza. In: DBI, 66, 2007, p. 450-452.

CAPESTRANO, Giovanni. Vita Sancti Bernardini Senensis per B. Iohannem a Capistrano conscripta. In: DE LA HAYE, Jean (ed.). Sancti Bernardini Senensis opera omnia. Paris, 1635, Vol. I, p. XXVII-XL.

CHECCOLI, Ippolita. The Vitae of Leading Italian Preachers of the Franciscan Observance: Fifteenth- and Sixteenth-Century Hagiographical Constructions. In: Franciscan

Studies, $n^{\circ}$ 71, 2013, p. 281-295.

CREMASCOLI, Giuseppe \& DONINI, Mauro (ed.). Gli statuti dell'Ospedale di Lodi (1466), Lodi: 1998.

DE GAIFFIER, Baudouin. Le vie de S. Bernardin du manuscrit de Rouge-Cloitre. In: Analecta Bollandiana, $\mathrm{n}^{\circ} 71,1953$, p. 282-322.

DE LAUDE MARTYRII = PSEUDO-CIPRIANO. De laude martyrii. In: Patrologia Latina, vol. 4, col. 787-804.

DELCORNO, Pietro. 'O felix adulescentia Bernardini! O ardentissima caritas cordis eius!': San Bernardino da Siena come modello per i laici. In: LOMBARDO, Eleonora (dir.).

Models of Virtues. The Role of Virtues in Sermons and Hagiography for new Saints' Cults (13th to 15th Century). Padova: Centro Studi Antoniani, 2016, p. 225-246.,

DIZIONARIO BIOGRAFICO DEGLI ITALIANI. Roma: Istituto della Enciclopedia Italiana, 1960- .

EVANGELISTI, Paolo. 'Dunque non sognate, fate fatti non solo parole': Bernardino de Siena e a proposta franciscana de uma religião civil. In: Varia Historia, $\mathrm{n}^{\circ} 31 / 55,2015, \mathrm{p}$. 81-126. 
EVANGELISTI, Paolo. La caritas cristomimetica francescana come strumento di costruzione della credibilità politico-economica (XIII-XV secolo). In: BOSCHIERO, Gemma (dir.) \& MOLINA, Barbara (dir.). Politiche del credito: Investimento, consumo, solidarietà. Asti: Centro Studi sui Lombardi, 2004, p. 84-112.

EVANGELISTI, Paolo. Metafore cristologiche per l'etica politica. Fonti e percorsi di ricerca nei testi di Giovanni da Capestrano. In: CACIOTTI \& MELLI, 2008, p. 141-168.

FARAONI, Monja. Antico tempio maestoso. La chiesa di San Francesco di Lodi. Bergamo: Bolis 2011.

FRANK, Thomas. Confraternite e assistenza. In: GAZZINI, Marina (dir.), Studi confraternali: orientamenti, problemi, testimonianze. Firenze: Firenza University Press, 2009, p. 217-238.

FRANK, Thomas. Heilsame Wortgefechte: Reformen europäischer Hospitäler vom 14. bis 16. Jahrhundert. Göttingen, V\&R Unipress, 2014.

GATTI PERER, Maria Luisa \& MAGGIONI, Chiara. L’immagine della carità nella Lombardia del Quattrocento. In: ALBERZONI, Maria Pia (dir.) \& GRASSI, Onorato (dir.), La carità a Milano nei secoli XII-XV. Milano: Jaca Book, 1989, p. 153-166.

GORINI, Raffaella. L'Ospedale di Santo Spirito della Carità a Lodi. Storia della fabbrica. In: Artes, $n^{\circ} 4,1996$, p. 44-53.

GORINI, Raffaella. L’Ospedale di Santo Spirito della Carità. In: MARUBBI, 1998, p. 51-53.

GRANATA, Elena. Insediamenti e conventi francescani a Lodi. In: Il francescanesimo in Lombardia: Storia e arte. Milano: Silvana, 1983, p. 331-343.

HANSKA, Jussi. Strategies of Sanity and Survival: Religious Responses to Natural Disasters in the Middle Ages. Helsinki: Finnish Literature Society, 2002.

I FRATI OSSERVANTI E LA SOCIETÀ IN ITALIA NEL SECOLO XV (Atti del XL Convegno internazionale, Assisi - Perugia, 11-13 ottobre 2012). Spoleto: CISAM, 2013.

JOHNSON, Timothy. Choir Prayers as the Place of Formation and Identity Definition: The Example of the Minorite Order. In Miscellanea Franciscana, ${ }^{\circ}$ 111, 2011, p. 123-137.

LEGENDA DE SANCTO BERNARDINO. Venezia: Simone de Luere, 1513.

LEVEROTTI, Franca. Ricerche sulle origini dell'ospedale Maggiore di Milano. In: Archivio Storico Lombardo, $\mathrm{n}^{\circ}$. 107, 1981, p.77-113.

LUDOVICO DA VICENZA. Vita post corporis translationem composita. In: Acta Sanctorum Maii, Paris: 1866, V, p. 93-107. 
MAFFEO VEGIO. De vita e obitu beati Bernardini. In: SOLVI, Daniele. L'agiografia su Bernardino santo (1450-146o). Firenze: Sismel - Galluzzo, 2014, p. 141-253.

MARUBBI, Mario (dir). L'oro e la porpora: Le arti a Lodi nel tempo del vescovo Pallavicino, 1456-1497. Cinisello Balsamo: Silvana, 1998.

MATTIOLI ROSSI, Laura. L'iconografia di s. Bernardino da Siena in Lombardia dal XV al XVIII secolo. In: Il francescanesimo in Lombardia: Storia e arte. Milano: Silvana, 1983, p. 232-246.

MERLO, Grado Giovanni. Nel nome di san Francesco: Storia dei frati Minori e del francescanesimo sino agli inizi del XVI secolo. Padova: EFR, 2003.

MIATELLO, André Luis Pereira. Santos e pregatores nas cidades medievais italianas: Retórica cívica e hagiografia. Belo Horizonte: Fino Traço, 2013.

MIXSON, James (dir.) \& ROEST, Bert (dir.). A Companion to Observant Reform in the Late Middle Ages and Beyond. Leiden: Brill, 2015.

MONTESANO, Marina. La memoria dell'esperienza di Bernardino da Siena nell'agiografia del XV secolo. In: Hagiographica, $n^{\circ}$ 1, 1994, p. 271-286.

NORMAN, Diana. "Santi cittadini": Vecchietta and the Civic Pantheon in Mid-FifteenthCentury Siena. In: SMITH, Timothy B. (dir.) \& STEINHOFF, Judith B. (dir.). Art as Politics in Late Medieval and Renaissance Siena. Farnham: Ashgate, 2012, p. 115140 .

PELLEGRINI, Letizia. Il processo di canonizzazione di Bernardino da Siena. Roma: Aracne, 2004.

PELLEGRINI, Letizia. Introduzione. In: Processo, 2009, p. $27^{*}-133^{*}$.

PELLEGRINI, Letizia. Nel cono d'ombra del Trecento: Pietà e devozione nella Siena di Bernardino. In: Beata Civitas: Pubblica pietà e devozioni private nella Siena del '300 (Siena 28-30 ottobre 2010). Firenze, Sismel - Galluzzo, 2016, p. 375-392.

PIANA, Celestino. Documenti intorno alla vita di S. Bernardino da Siena e codici delle sue opere. In: Bullettino storico bernardiniano, $n^{\circ}$. 10, 1944-50, p. 159-174.

PICCINNI, Gabriella. La strada come affare. Sosta, identificazione e depositi di denaro di pellegrini (1382-1446). In: PICCINNI, Gabriella \& TRAVAINI, Lucia. Il Libro del pellegrino (Siena 1382-1446): Affari, uomini, monete nell'Ospedale di S. Maria della Scala. Napoli, Liguori, 2003, p. 1-81.

PORTICI, Petra. Religiosità a Siena durante il primo Rinascimento: San Bernardino e gli affreschi del Pellegrinaio. In: NEVOLA, Fabrizio (dir.). Pio II Piccolomini: Il Papa del Rinascimento a Siena. Siena: Protagon, 2009, p. 51-78. 
PROCESSO = PELLEGRINI, Letizia (ed.). Il processo di canonizzazione di

Bernardino da Siena (1445-1450). Grottaferrata: Quaracchi, 2009.

PRUNAI, Giulio. Benvoglienti Leonardo. In: DBI, 8, 1966, p. 703-705.

QUAGLIONI, Diego. Un giurista sul pulpito. Giovanni da Capestrano predicatore e canonista. In: PÁSZTOR, Edith (dir.) \& PÁSZTOR, Lajos (dir.). S. Giovanni da

Capestrano nella Chiesa e nella società del suo tempo (Capestrano, 8 - 12 ottobre 1986). L’Aquila: 1989, p. 125-139.

RUSCONI, Roberto. Carcano Michele. In: DBI, 19, 1976, p. 742-744.

SERPICO, Fulvia (dir.). Biografia e agiografia di San Giacomo della Marca (Monteprandone, 29 novembre 2008). Firenze: Firenze: Sismel - Galluzzo, 2009.

SOLVI, Daniele. Agiografi e agiografie dell'Osservanza minoritica cismontana. In: SERPICO, 2009, p. 107-123.

SOLVI, Daniele. L'agiografia su Bernardino santo (1450-1460). Firenze: Sismel Galluzzo, 2014.

SORDINI, Beatrice. Il pellegrinaio degli uomini e gli ambienti sacri dell'Ospedale di Santa Maria della Scala di Siena: Spazi dell'assistenza, della rappresentanza e della propaganda (secoli XIV e XV). In: CARBONI, Mauro (dir.) \& MUZZARELLI, Maria Giuseppina (dir.), L'iconografia della solidarietà: La mediazione delle immagini (secoli XIII-XVIII). Venezia: Marsilio, 2011, p. 163-182.

VAUCHEZ, André. Saints admirables et saints imitables: les fonctions de l'hagiographie ont-elles changé aux derniers siècles du Moyen Âge. In: Les fonctions des saints dans le monde occidental (IIIe - XIIIe siècle). Roma: École Française de Rome, 1991, p. 161-172.

VITA ‘CLEMENTISSIMUS’, in: SOLVI, 2014, p. 69-139 